\title{
Application of hierarchical matrices to boundary element methods for elastodynamics based on Green's functions for a horizontally layered halfspace
}

\author{
P. Coulier*, S. François, G. Lombaert, G. Degrande \\ KU Leuven, Department of Civil Engineering, Kasteelpark Arenberg 40, B-3001 Leuven, Belgium
}

\begin{abstract}
This paper presents the application of hierarchical matrices to boundary element methods for elastodynamics based on Green's functions for a horizontally layered halfspace. These Green's functions are computed by means of the direct stiffness method; their application avoids meshing of the free surface and the layer interfaces. The effectiveness of the methodology is demonstrated through numerical examples, indicating that a significant reduction of memory and CPU time can be achieved with respect to the classical boundary element method. This allows increasing the problem size by one order of magnitude. The proposed methodology therefore offers perspectives to study large scale problems involving three-dimensional elastodynamic wave propagation in a layered halfspace, with possible applications in seismology and dynamic soil-structure interaction.
\end{abstract}

Keywords: boundary element method, elastodynamics, $\mathscr{H}$-matrices, halfspace Green's functions, railway induced vibrations.

\section{Introduction}

The boundary element (BE) method is well-suited to model three-dimensional (3D) elastodynamic wave propagation in a stratified halfspace, as the radiation of waves towards infinity is inherently taken into account. Moreover, a reduction of the spatial problem dimension is obtained due to the fact that only the boundaries of the domain have to be discretized. The BE method is therefore often employed to model a variety of problems, such as seismic site effects [1], railway induced vibrations [2] and other applications involving dynamic soil-structure interaction [3]. Accounting for the soil stratification is important in many of these applications, especially in presence of soft top layers. Although it is common to use closed form full space Green's functions in the BE formulation, numerically computed Green's functions for a horizontally layered halfspace can be incorporated as well in order to avoid meshing of the free surface and the layer interfaces, as will be done throughout this paper.

The advantages of the BE method are partially negated, however, by the fact that dense, fully populated unsymmetric matrices arise from the formulation, resulting in stringent memory and CPU requirements. This hinders the applicability of the BE method to large scale problems. Several fast BE methods have been developed in the past decade to improve the computational efficiency, including the fast multipole method (FMM) [4], the panel clustering technique [5] and methods based on hierarchical matrices [6]. In the FMM, the Green's functions are reformulated using a multipole expansion, which has proven to be very efficient if analytical expressions of the Green's functions are available [7]. Existing FMM formulations for (visco-)elastodynamics are therefore based on closed form full space fundamental solutions $[8,9,10]$, while innovative methods based on Green's functions for a homogeneous halfspace are currently under

*Corresponding author. Phone: + 32163216 75. Fax: + 3216321988 .

Email address: pieter.coulier@bwk.kuleuven.be (P. Coulier) 
development [11]. The latter approach has the main disadvantage, however, that a considerable amount of boundary elements is required for the discretization of the free surface and the layer interfaces, limiting the actual problem size that can be treated. A complementary class of fast BE methods is based on hierarchical matrices in combination with efficient algorithms such as adaptive cross approximation. These methods essentially are algebraic tools to approximate the BE matrices $[12,13]$, providing an alternative to tackle problems for which analytical expressions of the Green's functions are not available.

Hierarchical matrices have been employed to solve elliptic Helmholtz [14], Laplace [15] and elastostatic Lamé problems $[16,17]$. Only a few authors, however, have considered the use of hierarchical matrices for elastodynamics. Messner et al. [18] present an accelerated time domain elastodynamic boundary element formulation in which the time dependent problem is transformed into a system of decoupled Laplace domain problems using the convolution quadrature method. Full space elastodynamic fundamental solutions are employed in this approach, necessitating the discretization of the free surface to model wave propagation in a halfspace. Benedetti et al. [19] consider hierarchical matrices to solve elastodynamic crack problems using a dual boundary element method formulated in the Laplace domain, while Milazzo et al. [20] apply the concept to anisotropic elastodynamics in the frequency domain.

The aim of this paper is to demonstrate that hierarchical matrices are also suited to model 3D viscoelastodynamic wave propagation in a stratified halfspace, incorporating Green's functions for a horizontally layered halfspace. As the methodology is formulated in the frequency domain, material damping is accounted for through the correspondence principle [21] and visco-elastodynamic problems can easily be treated. The text is organized as follows. The basic concepts of the classical BE method are summarized in section 2, while the application of hierarchical matrices is discussed in section 3. The computation of the Green's functions for a horizontally layered halfspace is also briefly addressed. The numerical implementation of the proposed methodology is validated in section 4 and the computational performance is assessed in terms of memory and CPU requirements. The applicability of the novel approach is finally illustrated in section 5 by means of a case study in which dynamic through-soil coupling of closely spaced buildings under the influence of an incident wavefield is investigated.

\section{The boundary element method for elastodynamics based on Green's functions for a hori- zontally layered halfspace}

\subsection{Boundary integral equation}

Consider a domain $\Omega \in \mathbb{R}^{3}$ with boundary $\Sigma$, characterized by a unit outward normal vector $\mathbf{n}$. The classical boundary integral equation relates the displacement $\hat{u}_{i}\left(\mathbf{x}^{\prime}, \omega\right)$ in a point $\mathbf{x}^{\prime}$ to the elastodynamic state on the boundary $\Sigma$ in the frequency domain [22]:

$$
\kappa \hat{u}_{i}\left(\mathbf{x}^{\prime}, \omega\right)=\int_{\Sigma}\left(\hat{u}_{i j}^{\mathrm{G}}\left(\mathbf{x}^{\prime}, \mathbf{x}, \omega\right) \hat{t}_{j}^{\mathbf{n}}(\mathbf{x}, \omega)-\hat{t}_{i j}^{\mathrm{Gn}}\left(\mathbf{x}^{\prime}, \mathbf{x}, \omega\right) \hat{u}_{j}(\mathbf{x}, \omega)\right) \mathrm{d} S
$$

where the presence of body forces is neglected. A hat above a variable denotes its representation in the frequency domain. The Green's displacements $\hat{u}_{i j}^{\mathrm{G}}\left(\mathbf{x}^{\prime}, \mathbf{x}, \omega\right)$ and tractions $\hat{t}_{i j}^{\mathrm{Gn}} \mathbf{n}_{\mathrm{s}}\left(\mathbf{x}^{\prime}, \mathbf{x}, \omega\right)$ correspond to the fundamental solutions at a receiver $\mathbf{x}$ in a direction $\mathbf{e}_{j}$ due to a unit time harmonic point load at a source $\mathbf{x}^{\prime}$ in a direction $\mathbf{e}_{i}$. Throughout this paper, Green's functions for a homogeneous full space as well as a horizontally layered halfspace will be employed; the use of the latter avoids the discretization of the free surface and the layer interfaces. No closed form expressions are available for these Green's functions for layered soils, however, which implies that they have to be computed numerically. This will briefly be addressed in subsection 2.3 .

In equation (1), $\kappa=1$ when the point $\mathbf{x}^{\prime}$ is located inside the domain $\Omega$ and $\kappa=0$ if the point $\mathbf{x}^{\prime}$ is located outside the domain $\Omega$. The boundary integral equation (1) does not hold for points $\mathbf{x}^{\prime}$ located on the boundary $\Sigma$ due to the singular behaviour of the Green's functions. A classical limiting procedure $[23,24]$ results in Somigliana's identity, involving the evaluation of Cauchy principal value (CPV) integrals of the strongly singular Green's tractions. In this paper, however, a regularized boundary integral equation is employed, in which the evaluation of CPV integrals is avoided [24, 25, 26]. The regularization procedure 
is based on the fact that the singularity of the static and dynamic Green's functions at the source point is similar. In case of an unbounded domain $\Omega$, the regularized boundary integral equation, which is valid for points $\mathbf{x}^{\prime}$ located on the boundary $\Sigma$, reads as follows [24]:

$$
\hat{u}_{i}\left(\mathbf{x}^{\prime}, \omega\right)-\int_{\Sigma} \hat{u}_{i j}^{\mathrm{G}}\left(\mathbf{x}^{\prime}, \mathbf{x}, \omega\right) \hat{t}_{j}^{\mathbf{n}}(\mathbf{x}, \omega) \mathrm{d} S+\int_{\Sigma}\left(\hat{t}_{i j}^{\mathrm{Gn}}\left(\mathbf{x}^{\prime}, \mathbf{x}, \omega\right) \hat{u}_{j}(\mathbf{x}, \omega)-t_{i j}^{\mathrm{Gn} s}\left(\mathbf{x}^{\prime}, \mathbf{x}\right) \hat{u}_{j}\left(\mathbf{x}^{\prime}, \omega\right)\right) \mathrm{d} S=0
$$

where $t_{i j}^{\text {Gns }}$ denotes the static Green's tractions. The integral free term $\hat{u}_{i}\left(\mathbf{x}^{\prime}, \omega\right)$ in equation $(2)$ vanishes for a bounded domain $\Omega$. The boundary integrals in equation (2) are at most weakly singular and are evaluated using classical Gaussian integration.

\subsection{Boundary element discretization}

The regularized boundary integral equation (2) is solved numerically by discretizing the boundary $\Sigma$ with an appropriate number of boundary elements, resulting in a boundary element system of equations. For an unbounded domain $\Omega$, the displacements $\underline{\hat{\mathbf{u}}}(\omega)$ and tractions $\underline{\hat{\mathbf{t}}}(\omega)$ at the collocation points are related as follows:

$$
[\widehat{\mathbf{T}}(\omega)+\mathbf{I}] \underline{\hat{\mathbf{u}}}(\omega)=\widehat{\mathbf{U}}(\omega) \underline{\hat{\mathbf{t}}}(\omega)
$$

The system matrices $\widehat{\mathbf{U}}(\omega)$ and $\widehat{\mathbf{T}}(\omega)$ are fully populated unsymmetric matrices, while I represents a unit matrix, corresponding to the integral free term in the boundary integral equation. The latter vanishes for a bounded domain. The computation of the system matrices $\widehat{\mathbf{U}}(\omega)$ and $\widehat{\mathbf{T}}(\omega)$ requires integration of the Green's functions $\hat{u}_{i j}^{\mathrm{G}}\left(\mathbf{x}^{\prime}, \mathbf{x}, \omega\right)$ and $\hat{t}_{i j}^{\mathrm{Gn}}\left(\mathbf{x}^{\prime}, \mathbf{x}, \omega\right)$ over the boundary $\Sigma$, respectively.

A quadratic amount of memory $\left(\mathcal{O}\left(N_{\text {DOF }}^{2}\right)\right)$ is required to store the system matrices $\widehat{\mathbf{U}}(\omega)$ and $\widehat{\mathbf{T}}(\omega)$, where $N_{\text {DOF }}$ represents the number of degrees of freedom in the BE model. Furthermore, solving equation (3) by means of direct numerical solvers such as LU-decomposition requires a cubic amount of numerical operations $\left(\mathcal{O}\left(N_{\text {DOF }}^{3}\right)\right)$. The application of the classical boundary element method is therefore presently limited to problems involving up to $\mathcal{O}\left(10^{4}\right)$ degrees of freedom [12].

\subsection{Green's functions for layered soils: the direct stiffness method}

As mentioned in subsection 2.1, Green's functions $\hat{u}_{i j}^{\mathrm{G}}\left(\mathbf{x}^{\prime}, \mathbf{x}, \omega\right)$ and $\hat{t}_{i j}^{\mathrm{Gn}}\left(\mathbf{x}^{\prime}, \mathbf{x}, \omega\right)$ for a horizontally layered halfspace are employed in the boundary element formulation. These Green's functions are computed with the direct stiffness method [27] using the MATLAB toolbox EDT 2.2 [28]. The direct stiffness method provides element stiffness matrices for homogeneous layers and a homogeneous halfspace, formulated in the frequency-wavenumber domain. The stiffness matrix of a horizontally layered halfspace is obtained from the assembly of the element stiffness matrices. Solving the corresponding set of equations provides the Green's functions in the frequency-wavenumber domain. An inverse transformation from the wavenumber to the spatial domain is subsequently performed to obtain the Green's functions in the frequency-spatial domain. This is obtained by a numerical transformation algorithm, developed by Talman [29] and improved by Schevenels et al. [28, 30]. The calculation of these Green's functions is computationally expensive, however, in particular when a large number of source/receiver depths is considered.

The global system size in the direct stiffness method grows for an increasing number of soil layers and the time required to compute the Green's functions for a stratified halfspace increases correspondingly [31]. In cases with a very large number of layers, other numerical methods such as the propagator matrix method [32, 33] may provide a more efficient alternative; these Green's functions can be incorporated straightforwardly in the boundary element formulation. Nonetheless, the direct stiffness method has some appealing advantages compared to the latter method, such as the fact that stiffness matrices are symmetric, involve half as many degrees of freedom as propagator matrices, and remain robust and stable for thick layers and/or high frequencies if limiting expressions are implemented; propagator matrices, on the other hand, contain terms of exponential growth that require special consideration and treatment $[34,35,36]$. The direct stiffness method is therefore employed throughout this paper; a more elaborate comparison of the direct stiffness and the propagator matrix method is given by Kausel [37]. 


\section{Application of hierarchical matrices}

The applicability of the classical boundary element method is limited due to stringent memory and CPU requirements. The use of hierarchical matrices ( $\mathscr{C}$-matrices) provides an elegant way to treat fully populated matrices with almost linear complexity [38]. In this section, the basic principles of the boundary element method based on $\mathscr{H}$-matrices are briefly summarized. The reader is referred to the literature $[6,13,38,39]$ for a detailed description of the methodology.

\subsection{Hierarchical matrix assembly}

A $\mathscr{H}$-matrix is a data-sparse representation of a matrix, consisting of a collection of block matrices of various sizes. The construction of a $\mathscr{H}$-matrix requires several steps. First, a hierarchical cluster tree is constructed based on the boundary element mesh. At the lowest level (i.e. level 0), the cluster consists of the complete BE mesh. Each cluster is recursively partitioned into two (more or less equal) sons. Several techniques are available in order to obtain a suitable cluster tree (e.g. nested dissection [40, 41], cardinality balanced clustering $[13], \ldots$ ). In the following, a clustering strategy based on principal component analysis (PCA) is employed [12]. In PCA, the eigenvectors of the covariance matrix of a cluster are first calculated. The eigenvector corresponding to the largest eigenvalue gives the main direction of the cluster considered. A separation plane orthogonal to the aforementioned eigenvector is drawn through the center of the cluster, dividing it in two (more or less equal) sons. This procedure can be recursively applied to every son, until the clusters contain less or equal than a prescribed number $N_{\min }$ of elements (or nodes).

Second, admissible cluster pairs $(X, Y)$ are identified, i.e. cluster subdomains which satisfy a geometric admissibility criterion such that the corresponding fundamental solutions are smooth $[12,42]$ :

$$
\min \{\operatorname{diam}(\mathrm{X}), \operatorname{diam}(\mathrm{Y})\}<\eta \operatorname{dist}(\mathrm{X}, \mathrm{Y}) \quad 0<\eta<1
$$

where $\operatorname{diam}(\mathrm{X})$ denotes the maximal extent of cluster $X$ and $\operatorname{dist}(\mathrm{X}, \mathrm{Y})$ is the minimal distance between clusters $X$ and $Y$. The parameters $N_{\min }$ and $\eta$ affect the number of admissible blocks and the quality of the approximation of the admissible pairs [20,39]. A trade-off between accuracy and efficiency should be made when selecting these parameters.

The identification of admissible and inadmissible cluster pairs finally allows for the approximation of the BE matrices $\widehat{\mathbf{T}}(\omega)$ and $\widehat{\mathbf{U}}(\omega)$ by their hierarchical representations $\widehat{\mathbf{T}}_{\mathscr{H}}(\omega)$ and $\widehat{\mathbf{U}}_{\mathscr{H}}(\omega)$, respectively. For an admissible cluster pair $(X, Y)$, the fundamental solutions are sufficiently smooth such that the corresponding block BE matrices $\widehat{\mathbf{T}}_{(X, Y)}(\omega) \in \mathbb{C}^{m \times n}$ and $\widehat{\mathbf{U}}_{(X, Y)}(\omega) \in \mathbb{C}^{m \times n}$ can be approximated by low rank approximations. $m$ and $n$ denote the number of degrees of freedom in clusters $X$ and $Y$, respectively. For example, the block matrix $\widehat{\mathbf{T}}_{(X, Y)}(\omega)$ is approximated by $\widetilde{\mathbf{T}}_{(X, Y)}(\omega)$ :

$$
\widehat{\mathbf{T}}_{(X, Y)}(\omega) \simeq \widetilde{\mathbf{T}}_{(X, Y)}(\omega)=\left(\widehat{\mathbf{V}}_{\mathbf{T}_{(X, Y)}}(\omega)\right)\left(\widehat{\mathbf{W}}_{\mathbf{T}_{(X, Y)}}(\omega)\right)^{\star}
$$

with $\widehat{\mathbf{V}}_{\mathbf{T}_{(X, Y)}}(\omega) \in \mathbb{C}^{m \times k}$ and $\widehat{\mathbf{W}}_{\mathbf{T}_{(X, Y)}}(\omega) \in \mathbb{C}^{n \times k}$ and where $\star$ indicates the complex conjugate. $k$ is the rank of the representation. For $k(m+n)<m n, \widetilde{\mathbf{T}}_{(X, Y)}(\omega)$ is called a low rank approximation of $\widehat{\mathbf{T}}_{(X, Y)}(\omega)$, as the memory storage can be reduced from $\mathcal{O}(m n)$ to $\mathcal{O}(k(m+n))$ by storing $\widehat{\mathbf{V}}_{\mathbf{T}_{(X, Y)}}(\omega)$ and $\widehat{\mathbf{W}}_{\mathbf{T}_{(X, Y)}}(\omega)$ instead of $\widehat{\mathbf{T}}_{(X, Y)}(\omega)$, which is linear in $m$ and $n$. The rank $k$ is determined such that the approximation $\widetilde{\widehat{T}}_{(X, Y)}(\omega)$ is accurate up to a prescribed relative accuracy $\varepsilon$ :

$$
\left\|\widehat{\mathbf{T}}_{(X, Y)}(\omega)-\widetilde{\mathbf{T}}_{(X, Y)}(\omega)\right\|_{\mathrm{F}} \leq \varepsilon\left\|\widehat{\mathbf{T}}_{(X, Y)}(\omega)\right\|_{\mathrm{F}}
$$

where $\|\diamond\|_{F}$ indicates the Frobenius norm of the matrix $\diamond$. The partially pivoted adaptive cross approximation (ACA) algorithm $[12,43]$ is employed to compute the matrices $\widehat{\mathbf{V}}_{\mathbf{T}_{(X, Y)}}(\omega)$ and $\widehat{\mathbf{W}}_{\mathbf{T}_{(X, Y)}}(\omega)$ for the low rank approximation defined in equation (5). This is an algebraic approximation technique; the algorithm adaptively calculates some of the rows and columns of the original block matrix to obtain an approximation 
from few of the original matrix entries. The algorithm stops if the prescribed accuracy $\varepsilon$ is attained; the stopping criterion defined in equation (6) can however not be employed, as the original matrix $\widehat{\mathbf{T}}_{(X, Y)}(\omega)$ is never generated completely. An intrinsic stopping criterion based on the variation of the Frobenius norm in consecutive approximations is therefore used. The amount of numerical operations required in the ACA algorithm is $\mathcal{O}\left(k^{2}(m+n)\right)$ [12]. One of the major advantages of applying the ACA algorithm to obtain low rank approximations of the BE block matrices corresponding to admissible cluster pairs is that it is an algebraic approach, implying that there is no need for (semi-)analytical expressions of the Green's functions $\hat{u}_{i j}^{\mathrm{G}}\left(\mathbf{x}^{\prime}, \mathbf{x}, \omega\right)$ and $\hat{t}_{i j}^{\mathrm{Gn}}\left(\mathbf{x}^{\prime}, \mathbf{x}, \omega\right)$. The incorporation of Green's functions for layered soils has not yet been attempted before, however. The memory storage for these block BE matrices can be reduced even further by means of appropriate recompression techniques [38, 44, 45]. In this paper, a procedure based on the singular value decomposition (SVD) of the low rank approximations is employed [44]. Introducing the QR-decompositions of the matrices $\widehat{\mathbf{V}}_{\mathbf{T}_{(X, Y)}}(\omega)=\widehat{\mathbf{Q}}_{\mathbf{V}} \widehat{\mathbf{R}}_{\mathbf{V}}$ and $\widehat{\mathbf{W}}_{\mathbf{T}_{(X, Y)}}(\omega)=\widehat{\mathbf{Q}}_{\mathbf{W}} \widehat{\mathbf{R}}_{\mathbf{W}}$ in equation (5) gives:

$$
\widetilde{\mathbf{T}}_{(X, Y)}(\omega)=\widehat{\mathbf{Q}}_{\mathbf{V}} \widehat{\mathbf{R}}_{\mathbf{V}} \widehat{\mathbf{R}}_{\mathbf{W}}^{\star} \widehat{\mathbf{Q}}_{\mathbf{W}}^{\star}
$$

These matrix decompositions are calculated using a Householder transformation [46]. The SVD of the outer product of the two upper triangular matrices $\widehat{\mathbf{R}}_{\mathbf{V}}$ and $\widehat{\mathbf{R}}_{\mathbf{W}}$ is subsequently computed:

$$
\widehat{\mathbf{R}}_{\mathbf{V}} \widehat{\mathbf{R}}_{\mathrm{W}}^{\star}=\widehat{\mathbf{U}}_{\mathbf{R}} \widehat{\Sigma} \widehat{\mathbf{V}}_{\mathbf{R}}^{\star}
$$

with $\widehat{\Sigma} \in \mathbb{R}^{k \times k}$ a diagonal matrix containing the singular values in descending order. Combining equations $(7)$ and $(8)$ allows to write $\widetilde{\widehat{T}}_{(X, Y)}(\omega)$ as:

$$
\widetilde{\mathbf{T}}_{(X, Y)}(\omega)=\left(\widehat{\mathbf{Q}}_{\mathbf{V}} \widehat{\mathbf{U}}_{\mathbf{R}}\right) \widehat{\Sigma}\left(\widehat{\mathbf{Q}}_{\mathbf{W}} \widehat{\mathbf{V}}_{\mathbf{R}}\right)^{\star}
$$

which can be identified as the SVD of $\widetilde{\mathbf{T}}_{(X, Y)}(\omega)$, as the matrices $\widehat{\mathbf{Q}}_{\mathbf{V}} \widehat{\mathbf{U}}_{\mathbf{R}}$ and $\widehat{\mathbf{Q}}_{\mathbf{W}} \widehat{\mathbf{V}}_{\mathbf{R}}$ are both unitary [45]. A memory reduction is then obtained by discarding the smallest singular values and corresponding singular vectors in equation (9), hence reducing the rank, while maintaining the desired approximation accuracy. This recompression procedure is applied to every low rank approximation immediately after its assembly through ACA and only requires a limited amount of additional numerical operations of $\mathcal{O}\left(k^{2}(m+n+k)\right)[45]$.

For cluster pairs $(X, Y)$ not satisfying the admissibility criterion (4), the fundamental solutions show a singular behaviour which does not allow constructing a low rank approximation of the corresponding block BE matrices. These blocks are therefore computed exactly.

\subsection{Solving the $\mathscr{H}-B E$ equations}

Following the procedure outlined above for every cluster pair $(X, Y)$ leads to an approximation of the BE matrices $\widehat{\mathbf{T}}(\omega)$ and $\widehat{\mathbf{U}}(\omega)$ by their hierarchical representations $\widehat{\mathbf{T}}_{\mathscr{H}}(\omega)$ and $\widehat{\mathbf{U}} \mathscr{H}(\omega)$, respectively, and the $\mathrm{BE}$ equation (3) can be replaced by:

$$
\left[\widehat{\mathbf{T}}_{\mathscr{H}}(\omega)+\mathbf{I}\right] \underline{\hat{\mathbf{u}}}(\omega)=\widehat{\mathbf{U}}_{\mathscr{H}}(\omega) \underline{\hat{\mathbf{t}}}(\omega)
$$

In order to solve equation (10), iterative Krylov subspace methods are well suited. The matrix-vector multiplication forms the core of iterative solvers and the complexity of this operation is only $\mathcal{O}\left(N_{\mathrm{DOF}} \log N_{\mathrm{DOF}}\right)$ for $\mathscr{H}$-matrices [13]. In this paper, all equations are therefore solved by means of the generalized minimal residual method (GMRES) [47]. As will be illustrated in subsection 4.2, the contribution of the time required for solving equation (10) to the total computation time is negligible compared to the assembly time of the $\mathscr{H}$-matrices; no preconditioner is therefore incorporated in the iterative solver. 


\section{Validation}

The BE method based on $\mathscr{H}$-matrices outlined in section 3 has been implemented in the MATLAB toolbox BEMFUN [48]. The core of this toolbox is implemented in $\mathrm{C}++$ using the MATLAB MEX interface in order to achieve both a seamless integration with MATLAB and a high numerical efficiency. In the following subsections, three examples are considered to validate the numerical implementation and to demonstrate the effectiveness of the methodology. The first example is included to validate the correct implementation of $\mathscr{H}$-matrix arithmetics and does not involve Green's functions for a layered halfspace, while the second and the third example focus on the application of the novel method, incorporating Green's functions of a layered and homogeneous halfspace, respectively. All calculations have been performed on Intel ${ }^{\circledR}$ Xeon ${ }^{\circledR}$ E5520 (2.26 GHz) CPUs.

\subsection{D spherical cavity subjected to an internal pressure}

A 3D spherical cavity with radius $r_{0}=1 \mathrm{~m}$ in a full space loaded by an internal pressure $\hat{p}(\omega)=1 \mathrm{~Pa} / \mathrm{Hz}$ is investigated in this subsection. The full space is characterized by a shear wave velocity $C_{\mathrm{s}}=150 \mathrm{~m} / \mathrm{s}$, a dilatational wave velocity $C_{\mathrm{p}}=300 \mathrm{~m} / \mathrm{s}$ and a density $\rho=1800 \mathrm{~kg} / \mathrm{m}^{3}$. No material damping is taken into account.

The unit sphere is discretized by means of 3072 eight node quadrilateral boundary elements with element collocation. Both the classical and hierarchical BE method are used to calculate the response in a frequency range between $0 \mathrm{~Hz}$ and $100 \mathrm{~Hz}$, where analytical full space fundamental solutions [23, 37] are employed. For the latter method, a hierarchical cluster tree is constructed based on the elements' center, as an element collocation scheme is used. A minimum number of elements $N_{\min }=24$ is specified, resulting in $\log _{2}(3072 / 24)=7$ cluster levels. Figure 1 shows the hierarchical block structure of the matrices of $\widehat{\mathbf{T}}_{\mathscr{H}}(\omega)$ and $\widehat{\mathbf{U}}_{\mathscr{H}}(\omega)$ arising from this hierarchical clustering, where a value of 0.95 is attributed to the parameter $\eta$ in the admissibility criterion (4). A threshold $\varepsilon=10^{-3}$ has been used in the ACA algorithm to obtain low rank approximations of the blocks corresponding to admissible cluster pairs, while a tolerance of $10^{-4}$ was specified in the iterative GRMES solver.

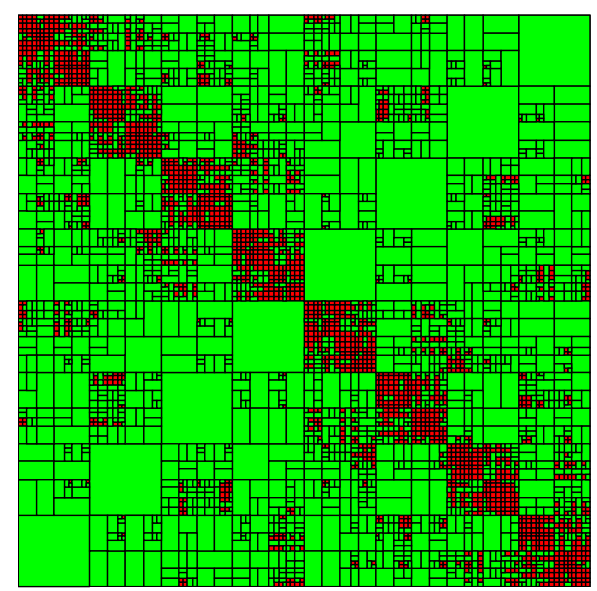

Figure 1: Hierarchical matrix decomposition corresponding to the BE model of a spherical cavity. The green blocks corresponding to admissible cluster pairs are approximated by means of ACA, while the red blocks corresponding to inadmissible cluster pairs are computed exactly.

Figure 2 shows the real and imaginary part of the radial displacement at the point $\left\{r=r_{0}, \theta=0, z=\right.$ $0 \mathrm{~m}$ \}, calculated with the classical and the hierarchical BE method, respectively. A perfect agreement between the results of both methods can be observed. These results are furthermore compared to the 
analytical solution for the radial displacement in the full space, defined as [37]:

$$
\hat{u}_{r}(r, \omega)=\frac{r_{0}^{3}}{4 \rho C_{\mathrm{s}}^{2} r^{2}} \frac{1+i \omega_{\mathrm{p}}}{1+i \omega_{0}-\left(\omega_{0} \frac{C_{\mathrm{p}}}{2 C_{\mathrm{s}}}\right)^{2}} \exp \left(-i\left(\frac{r}{r_{0}}-1\right) \omega_{0}\right)
$$

where $\omega_{\mathrm{p}}=\omega r / C_{\mathrm{p}}$ and $\omega_{0}=\omega r_{0} / C_{\mathrm{p}}$. The results of both numerical methods agree very well with the analytical solution (i.e. equation (11) evaluated for $r=r_{0}$ ) in the low frequency range, with some small deviations above $40 \mathrm{~Hz}$.
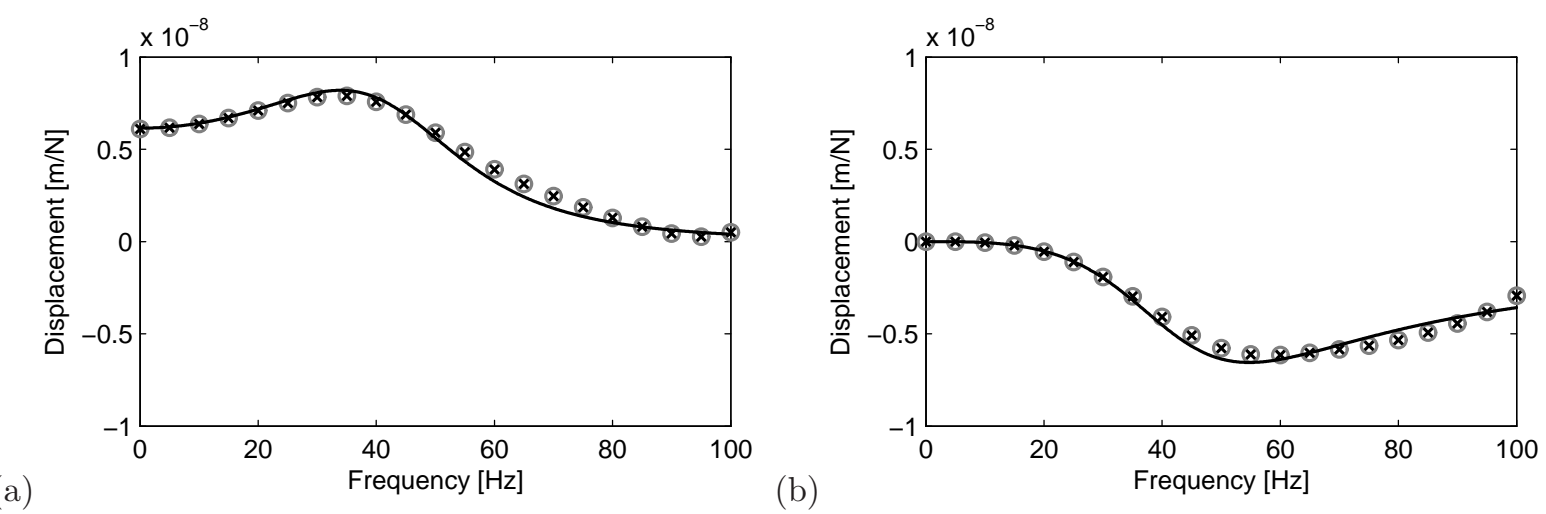

Figure 2: (a) Real and (b) imaginary part of the radial displacement at $\left\{r=r_{0}, \theta=0, z=0 \mathrm{~m}\right\}$ due to a unit harmonic pressure applied in a spherical cavity with $r_{0}=1 \mathrm{~m}$. The solution obtained with the hierarchical BE method (grey circles) is compared to the solution obtained with the classical BE method (black crosses) and the analytical solution (solid line) [37].

The integral representation theorem subsequently allows for the computation of the radiated wavefield in the soil from the displacements and tractions on the boundary. Figure 3 shows the real and imaginary part of the radial displacement at the point $\{r=10 \mathrm{~m}, \theta=0, z=0 \mathrm{~m}\}$. The solutions of the classical and hierarchical $\mathrm{BE}$ method are clearly in good correspondence and agree with the analytical solution (i.e. equation (11) evaluated for $r=10 \mathrm{~m}$ ).
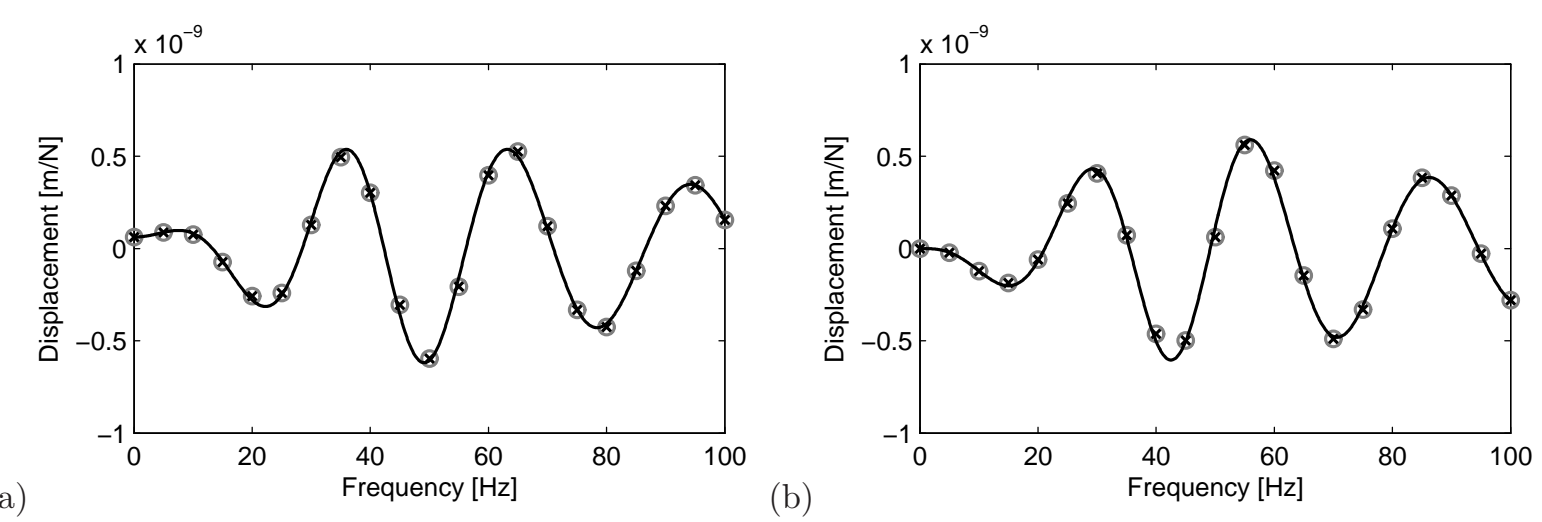

Figure 3: (a) Real and (b) imaginary part of the radial displacement at $\{r=10 \mathrm{~m}, \theta=0, z=0 \mathrm{~m}\}$ due to a unit harmonic pressure applied in a spherical cavity with $r_{0}=1 \mathrm{~m}$. The solution obtained with the hierarchical BE method (grey circles) is compared to the solution obtained with the classical BE method (black crosses) and the analytical solution (solid line) [37].

The accuracy of the BE method based on $\mathscr{H}$-matrices is investigated in more detail in figure 4 , showing the relative error $\left\|\hat{u}_{r, \mathscr{H}}\left(r_{0}, \omega\right)-\hat{u}_{r, \mathrm{c}}\left(r_{0}, \omega\right)\right\| /\left\|\hat{u}_{r, \mathrm{c}}\left(r_{0}, \omega\right)\right\| . \hat{u}_{r, \mathscr{H}}\left(r_{0}, \omega\right)$ and $\hat{u}_{r, \mathrm{c}}\left(r_{0}, \omega\right)$ represent the radial 
displacement on the boundary of the cavity, calculated with the hierarchical and the classical BE method, respectively. Although a threshold $\varepsilon=10^{-3}$ is prescribed in the ACA algorithm for the approximation of the matrix blocks corresponding to admissible cluster pairs, the overall computational precision is $\mathcal{O}\left(10^{-4}\right)$, except at high frequencies.

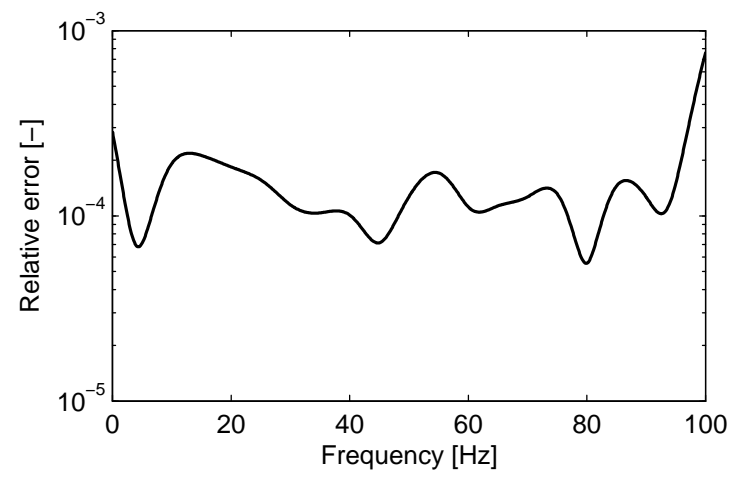

Figure 4: Relative error $\left\|\hat{u}_{r, \mathscr{H}}\left(r_{0}, \omega\right)-\hat{u}_{r, \mathrm{c}}\left(r_{0}, \omega\right)\right\| /\left\|\hat{u}_{r, \mathrm{c}}\left(r_{0}, \omega\right)\right\|$ on the boundary of a spherical cavity with $r_{0}=1 \mathrm{~m}$.

\subsection{Impedance of a massless rigid square surface foundation on a horizontally layered halfspace}

A massless rigid square surface foundation resting on a horizontally layered halfspace is considered in this subsection. The foundation side equals $d=5 \mathrm{~m}$. The soil consists of two layers on a halfspace, each with a thickness of $2 \mathrm{~m}$. The shear wave velocity $C_{\mathrm{s}}$ is equal to $150 \mathrm{~m} / \mathrm{s}$ in the top layer, $250 \mathrm{~m} / \mathrm{s}$ in the second layer, and $300 \mathrm{~m} / \mathrm{s}$ in the underlying halfspace. The Poisson's ratio $\nu$ is $1 / 3$ everywhere, resulting in dilatational wave velocities $C_{\mathrm{p}}$ of $300 \mathrm{~m} / \mathrm{s}, 500 \mathrm{~m} / \mathrm{s}$, and $600 \mathrm{~m} / \mathrm{s}$, respectively. Material damping ratios $\beta_{\mathrm{s}}=\beta_{\mathrm{p}}=0.025$ in both deviatoric and volumetric deformation are attributed to the layers and the halfspace, while a uniform density $\rho=1800 \mathrm{~kg} / \mathrm{m}^{3}$ is considered throughout the medium.

The classical as well as the hierarchical BE method are employed to calculate the vertical soil impedance $\hat{K}_{z z}^{\mathrm{s}}(\omega)$, defined as:

$$
\hat{K}_{z z}^{\mathrm{s}}(\omega)=\int_{\Sigma} \boldsymbol{\psi}_{z z} \cdot \hat{\mathbf{t}}_{\mathrm{s}}^{\mathbf{n}_{\mathrm{s}}}\left(\hat{\mathbf{u}}_{\mathrm{sc}}\left(\boldsymbol{\psi}_{z z}\right) \mathrm{d} S\right.
$$

where $\boldsymbol{\psi}_{z z}$ indicates the vertical rigid body translation of the foundation. The soil-structure interface $\Sigma$ is discretized by means of four node quadrilateral boundary elements with element collocation. The tractions $\hat{\mathbf{t}}_{\mathrm{s}}^{\mathbf{n}_{\mathrm{s}}}\left(\hat{\mathbf{u}}_{\mathrm{sc}}\left(\boldsymbol{\psi}_{z z}\right)\right.$ due to imposed displacements $\boldsymbol{\psi}_{z z}$ are obtained by solving equations (3) and (10), respectively. As a surface foundation is considered, the system matrices $\widehat{\mathbf{T}}(\omega)$ and $\widehat{\mathbf{T}} \mathscr{H}(\omega)$ are zero. The same values for the parameters $\eta, N_{\min }, \varepsilon$ and the tolerance in the GMRES solver as specified in subsection 4.1 are used in the BE method based on $\mathscr{H}$-matrices. As mentioned in section 2, Green's functions for a layered halfspace are incorporated in both $\mathrm{BE}$ formulations [27, 28], avoiding the necessity to discretize the free surface and the layer interfaces.

The vertical soil impedance $\hat{K}_{z z}^{\mathrm{s}}(\omega)$ can alternatively be written in the following form [49]:

$$
\hat{K}_{z z}^{\mathrm{s}}(\omega)=K_{z z 0}^{\mathrm{s}}\left(k_{z z}\left(a_{0}\right)+i a_{0} c_{z z}\left(a_{0}\right)\right)
$$

where $a_{0}=\omega B / C_{\mathrm{s}}$ is a dimensionless frequency and $B=d / 2$ a characteristic length of the foundation. $K_{z z 0}^{\mathrm{s}}$ indicates the static stiffness, while $k_{z z}\left(a_{0}\right)$ and $c_{z z}\left(a_{0}\right)$ are dimensionless stiffness and damping coefficients, respectively. A BE mesh consisting of $30 \times 30$ equally sized elements is used. Up to nine elements per shear wavelength $\lambda_{\mathrm{s}}=C_{\mathrm{s}} / f=2 \pi B / a_{0}$ are provided at the maximum dimensionless frequency of 10 (determined by the shear wave velocity of the top layer). Figure 5 shows the coefficients $k_{z z}\left(a_{0}\right)$ and $c_{z z}\left(a_{0}\right)$ in a 

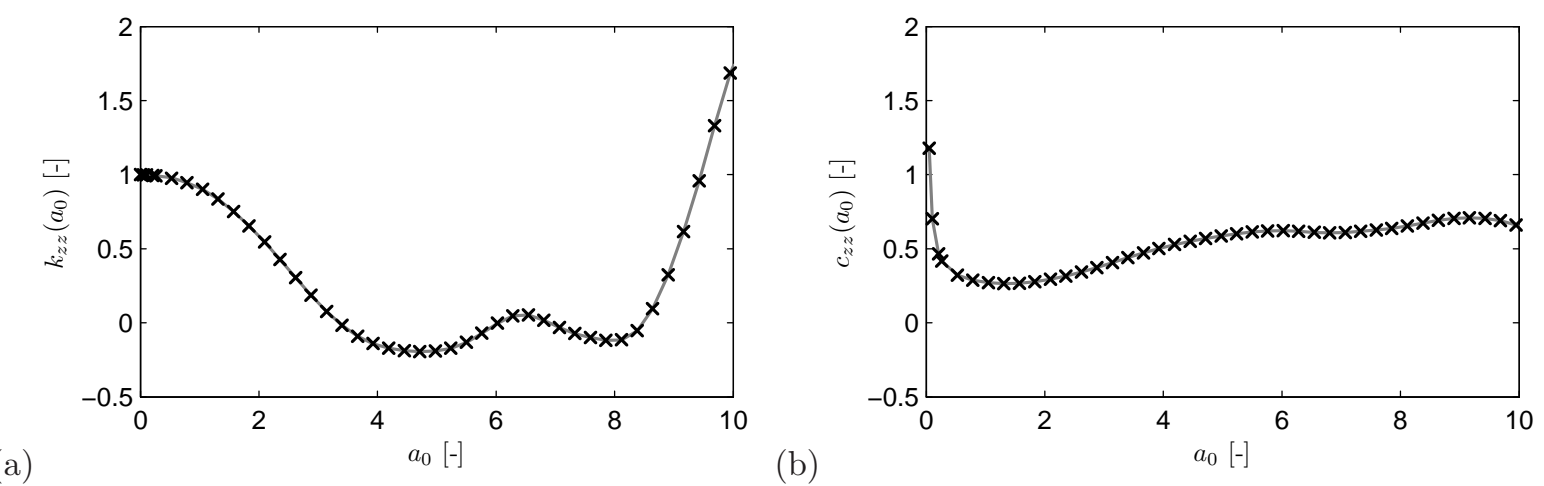

Figure 5: (a) Dimensionless stiffness coefficient $k_{z z}\left(a_{0}\right)$ and (b) damping coefficient $c_{z z}\left(a_{0}\right)$ of a massless rigid square surface foundation on a layered halfspace in function of the dimensionless frequency $a_{0}$. The solution obtained with the hierarchical BE method (grey line) is compared to the solution obtained with the classical BE method (black crosses).

dimensionless frequency range between 0 and 10. A perfect match between the classical and hierarchical BE method can be observed.

The influence of the threshold $\varepsilon$ used in the ACA algorithm on the accuracy and efficiency of the BE method based on $\mathscr{H}$-matrices is investigated in figures $6-8$. The value of the threshold $\varepsilon$ is varied logarithmically between $10^{-1}$ and $10^{-4}$. It is clearly illustrated in figure 6 that $\varepsilon$ should be smaller than $10^{-2}$ in order to obtain reliable results. The accuracy of the proposed methodology is also assessed in figure 7, which shows the relative error $\left\|\hat{K}_{z z, \mathscr{H}}^{\mathrm{s}}\left(a_{0}\right)-\hat{K}_{z z, \mathrm{c}}^{\mathrm{s}}\left(a_{0}\right)\right\| /\left\|\hat{K}_{z z, \mathrm{c}}^{\mathrm{s}}\left(a_{0}\right)\right\|$, where $\hat{K}_{z z, \mathscr{H}}^{\mathrm{s}}\left(a_{0}\right)$ and $\hat{K}_{z z, \mathrm{c}}^{\mathrm{s}}\left(a_{0}\right)$ represent the vertical soil impedance computed with the hierarchical and the classical BE method, respectively. The relative error considerably decreases for reduced values of $\varepsilon$. Furthermore, a decrease of almost one order of magnitude is observed for increasing dimensionless frequencies in case $\varepsilon=10^{-1}$ and $\varepsilon=10^{-2}$, while the relative error is less dependent on the frequency for the other cases. The RAM memory usage with respect to the classical BE method is shown in figure 8. As expected, the efficiency decreases for reduced values of $\varepsilon$, as well as for an increasing dimensionless frequency $a_{0}$. Such trends have also been observed in hierarchical BE methods for anisotropic elastodynamic problems [19, 20]. Figures 6-8 clearly indicate that the overall computational precision and the RAM memory usage are strongly determined by the value of the threshold $\varepsilon$. A trade-off between accuracy and efficiency should hence be made when applying the proposed methodology.
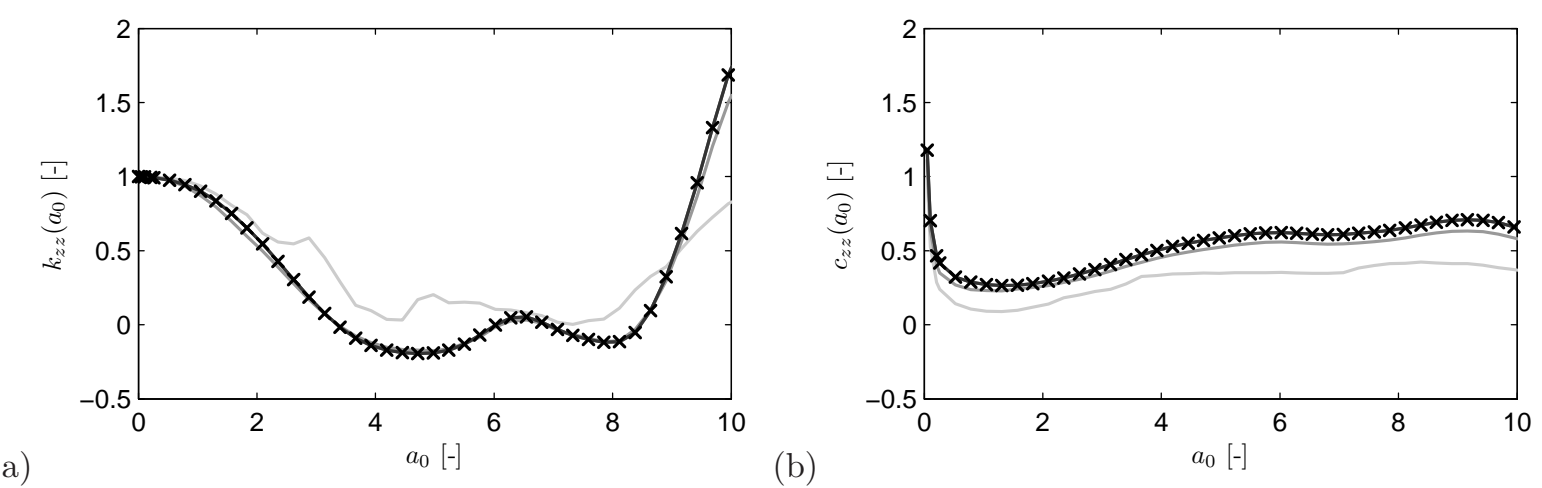

Figure 6: (a) Dimensionless stiffness coefficient $k_{z z}\left(a_{0}\right)$ and (b) damping coefficient $c_{z z}\left(a_{0}\right)$ of a massless rigid square surface foundation on a layered halfspace in function of the dimensionless frequency $a_{0}$. The solution obtained with the hierarchical BE method (solid lines) for the threshold $\varepsilon$ used in the ACA algorithm varying from $10^{-1}$ (light grey line) to $10^{-4}$ (dark grey line) is compared to the solution obtained with the classical BE method (black crosses). 


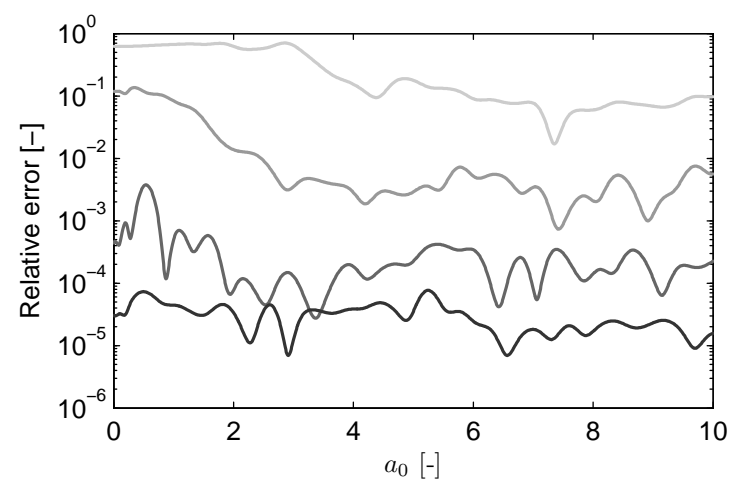

Figure 7: Relative error $\left\|\hat{K}_{z z, \mathscr{H}}^{\mathrm{s}}\left(a_{0}\right)-\hat{K}_{z z, \mathrm{c}}^{\mathrm{s}}\left(a_{0}\right)\right\| /\left\|\hat{K}_{z z, \mathrm{c}}^{\mathrm{s}}\left(a_{0}\right)\right\|$ in function of the dimensionless frequency $a_{0}$, for the threshold $\varepsilon$ used in the ACA algorithm varying from $10^{-1}$ (light grey line) to $10^{-4}$ (dark grey line).

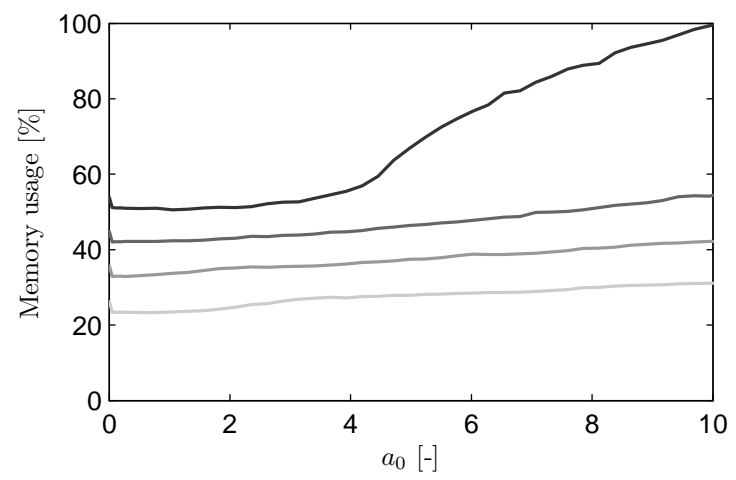

Figure 8: Memory usage with respect to the classical BE method in function of the dimensionless frequency $a_{0}$, for the threshold $\varepsilon$ used in the ACA algorithm varying from $10^{-1}$ (light grey line) to $10^{-4}$ (dark grey line).

The soil response due to a unit vertical rigid body translation $\psi_{z z}$ of the foundation is shown in figures $9 \mathrm{a}$ and $9 \mathrm{~b}$ at dimensionless frequencies $a_{0}=5$ and $a_{0}=10$, respectively; the classical and hierarchical BE approach yield exactly the same result. A relatively low frequency range is considered in this example, as the classical BE method is not well suited to provide accurate reference results at high frequencies within reasonable computation times. The BE method based on $\mathscr{H}$-matrices, however, is able to model high frequent wave propagation in a layered halfspace. The radiated wavefield at the surface of the soil is shown in figure $9 \mathrm{c}$ at a relatively high dimensionless frequency $a_{0}=50$. A BE mesh consisting of $100 \times 100$ equally sized elements is used for this calculation, providing six elements per shear wavelength $\lambda_{\mathrm{s}}=C_{\mathrm{s}} / f=2 \pi B / a_{0}$. The propagation of wave fronts parallel to the foundation edges can clearly be observed.

In order to demonstrate the effectiveness of the BE method based on $\mathscr{H}$-matrices, the vertical soil impedance $\hat{K}_{z z}^{\mathrm{s}}(\omega)$ is reconsidered at a particular dimensionless frequency $a_{0}=\pi$, for an increasing number of boundary elements. As an element collocation scheme is applied, the number of degrees of freedom equals three times the number of elements. The stiffness and damping coefficients $k_{z z}\left(a_{0}=\pi\right)$ and $c_{z z}\left(a_{0}=\pi\right)$ are shown as a function of the number of degrees of freedom in figure 10. The results of the hierarchical and classical BE method both converge to a value $\hat{K}_{z z}^{\mathrm{s}}=K_{z z 0}^{\mathrm{s}}\left(0.077+i a_{0} 0.416\right) \mathrm{N} / \mathrm{m}$ and are in good correspondence with each other. The application of the classical BE method is, however, limited to a model size of 43200 degrees of freedom, as the storage of the complex floating point entries of $\widehat{\mathbf{U}}(\omega)$ in double precision requires $2 \times N_{\text {DOF }}^{2} \times 8$ bytes $=27.8 \mathrm{~GB}$ of RAM memory, which is the limit of the hardware employed for the calculations presented in this paper. Figure 11a compares the memory required to store 

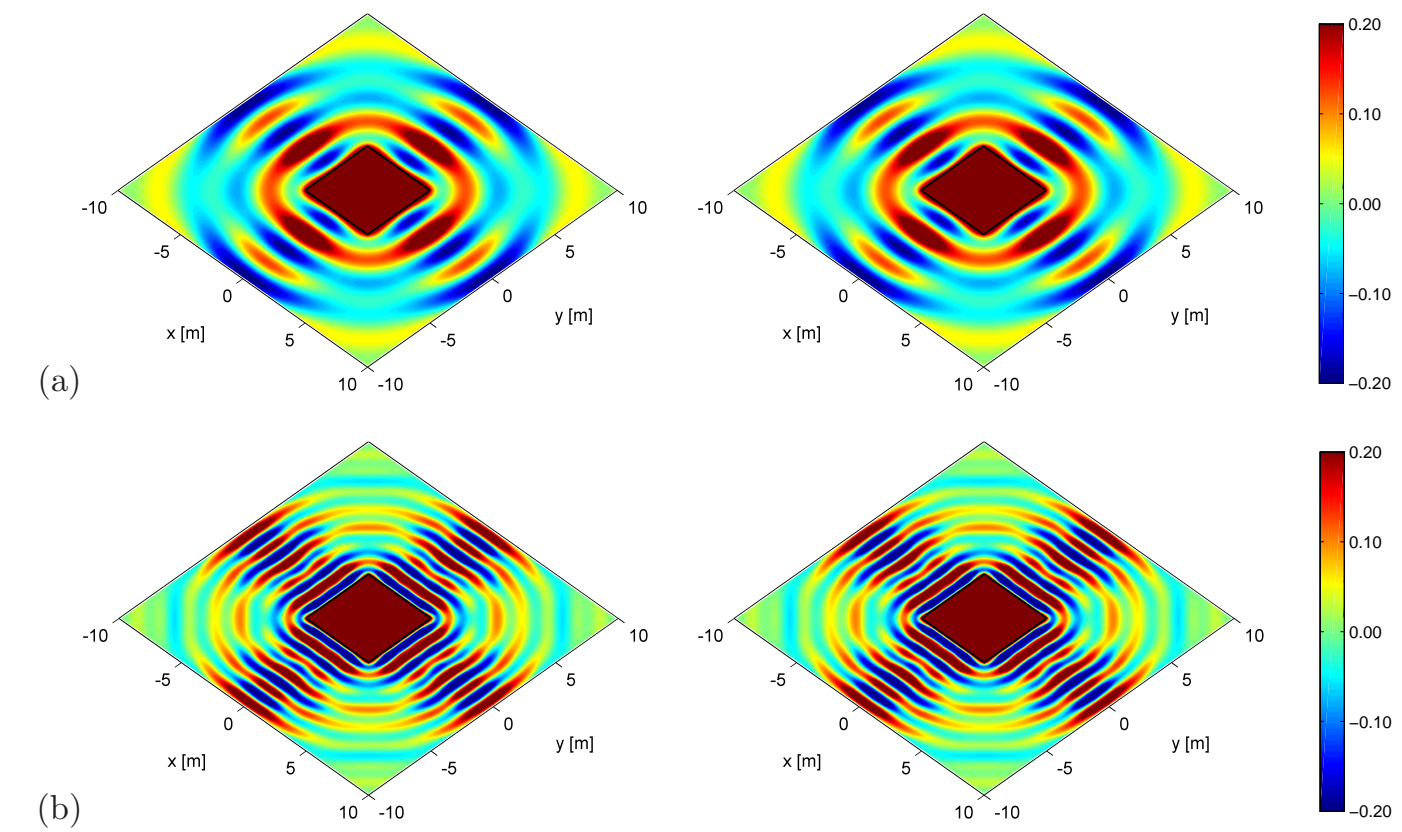

(c)

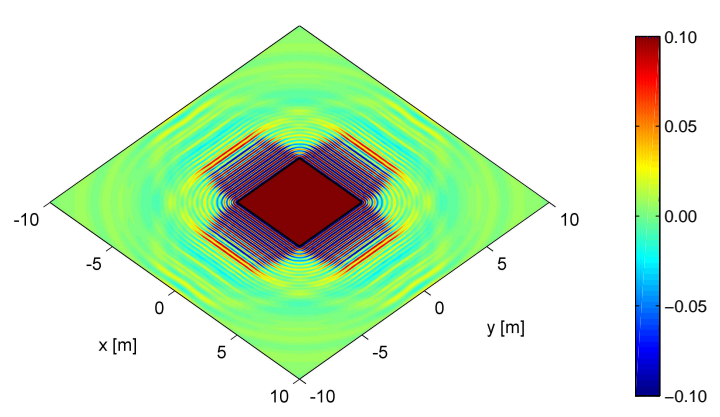

Figure 9: Real part of the vertical displacement $\hat{u}_{z}(\mathbf{x}, \omega)$ due to an imposed unit vertical rigid body translation $\boldsymbol{\psi}_{z z}$ of a massless rigid square surface foundation on a layered halfspace at a dimensionless frequency (a) $a_{0}=5$, (b) $a_{0}=10$ and (c) $a_{0}=50$, obtained with the classical (left) and the hierarchical (right) BE method. The classical BE method is not well suited to provide accurate reference results for (c) within reasonable computation times. 
the BE matrices $\widehat{\mathbf{U}}(\omega)$ and $\widehat{\mathbf{U}} \mathscr{H}(\omega)$ on a double logarithmic plot, in which the slope of the curve corresponds to the power relating the number of degrees of freedom and the required memory. As expected, a quadratic trend $\mathcal{O}\left(N_{\text {DOF }}^{2}\right)$ can be observed for the classical BE method. For the hierarchical BE method, however, the memory requirement is of the order $\mathcal{O}\left(N_{\mathrm{DOF}} \log _{10}^{3} N_{\mathrm{DOF}}\right)$, allowing for the extension of the model size up to 399675 degrees of freedom with $28 \mathrm{~GB}$ of RAM memory available. Applying the recompression procedure outlined in subsection 3.1 even allows to consider up to 468075 degrees of freedom with the same amount of memory. In comparison, $2380 \mathrm{~GB}$ and $3265 \mathrm{~GB}$ of RAM would, respectively, be required in order to handle such models with the classical BE method. Figure 11b shows the CPU time required to calculate $\hat{K}_{z z}^{\mathrm{s}}\left(a_{0}=\pi\right)$, including both the time to assemble the matrices $\widehat{\mathbf{U}}(\omega)$ or $\widehat{\mathbf{U}} \mathscr{H}(\omega)$ and to solve equation $(3)$ or (10), respectively. For relatively small models $\left(\mathcal{O}\left(10^{3}\right)\right)$, the classical BE method turns out to be a little faster, but the hierarchical approach is considerably more efficient from a moderate model size on.

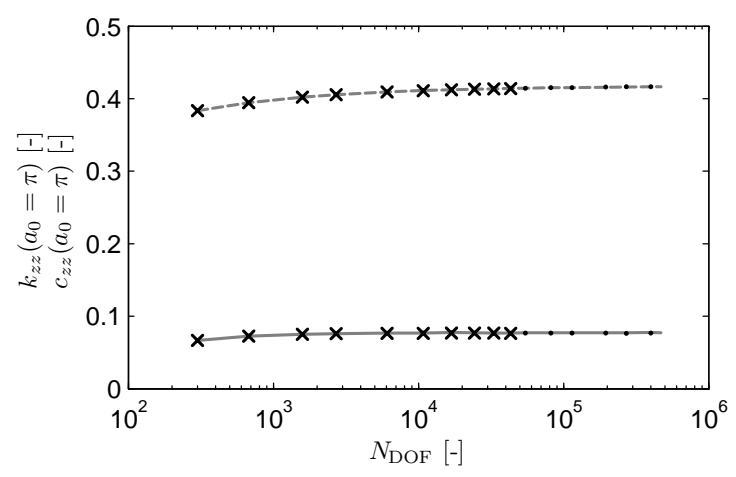

Figure 10: Dimensionless stiffness coefficient $k_{z z}\left(a_{0}=\pi\right)$ (solid line) and damping coefficient $c_{z z}\left(a_{0}=\pi\right)($ dashed line) of a massless rigid square surface foundation on a layered halfspace for an increasing number of degrees of freedom. The solution obtained with the hierarchical BE method with (grey lines) and without recompression (black dots) is compared to the solution obtained with the classical BE method (black crosses).
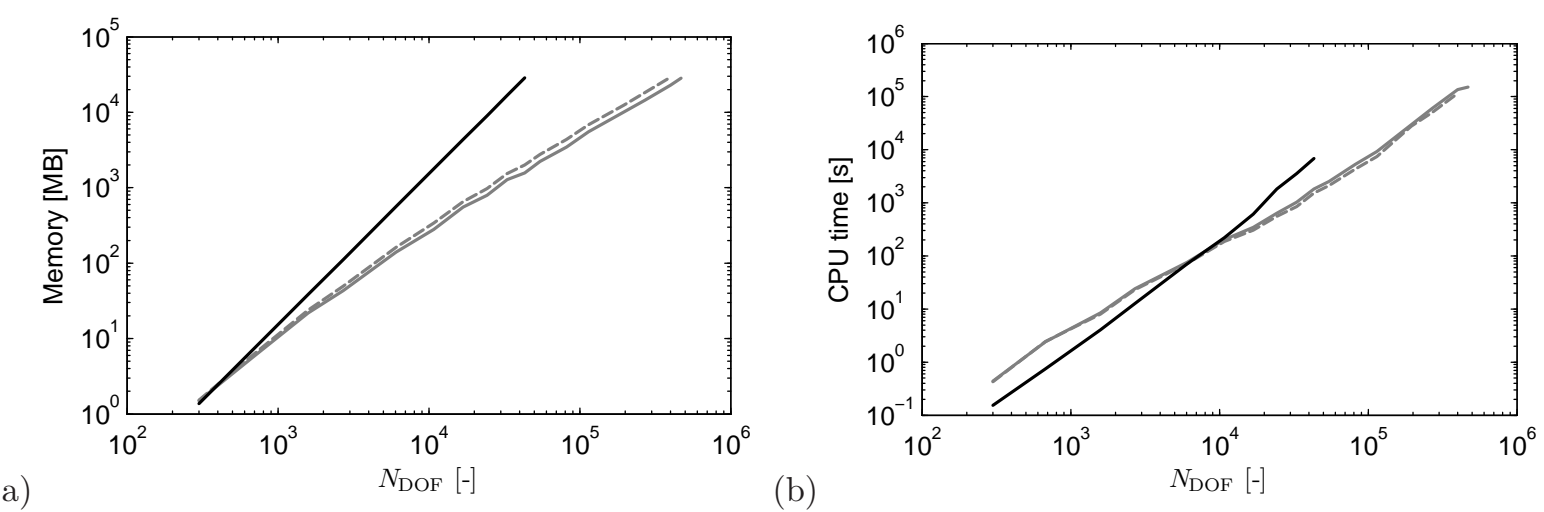

Figure 11: (a) RAM memory and (b) total CPU time required for an increasing number of degrees of freedom with the classical (black lines) and the hierarchical BE method with (solid grey lines) and without (dashed grey lines) recompression.

The contribution of the assembly and solution time to the total CPU time is further investigated in figure 12 (in case no recompression is applied). For the classical BE method, the assembly time shows a quadratic trend. The solution time of the direct solver, however, increases in a cubic way. The time required to solve equation (3) will therefore dominate the total solution time of the classical BE method for large models. Figure 12 also shows that the contribution of the solution time is negligible compared to the assembly time 
for the hierarchical BE method. No effort has therefore been made to incorporate a preconditioner in the iterative GMRES solver, such as an $\mathscr{H}-\mathrm{LU}$ preconditioner proposed in [50], as speeding up the iterative solver will not result in a significant reduction of the total CPU time required in the hierarchical BE method.

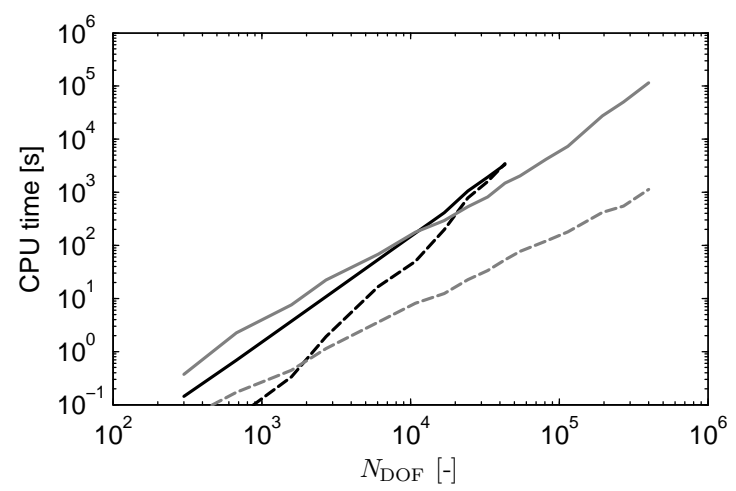

Figure 12: CPU time required to assemble the matrices $\widehat{\mathbf{U}}(\omega)$ or $\widehat{\mathbf{U}}_{\mathscr{H}}(\omega)$ (solid lines) and to solve equation (3) or (10) (dashed lines) with the classical (black) and the hierarchical (grey) BE method.

\subsection{Diffraction of a vertically incident plane $P$-wave by a semi-spherical cavity}

In this subsection, the diffraction of a vertically incident plane $\mathrm{P}$-wave by a semi-spherical cavity with radius $a$, representing a canyon, is investigated (figure 13). For validation purposes [51, 52, 53, 54], a homogeneous halfspace is considered, although the diffraction in a layered halfspace can be easily treated as well. The halfspace has a shear wave velocity $C_{\mathrm{s}}=150 \mathrm{~m} / \mathrm{s}$, a dilatational wave velocity $C_{\mathrm{p}}=259.8 \mathrm{~m} / \mathrm{s}$, a density $\rho=1800 \mathrm{~kg} / \mathrm{m}^{3}$ and a material damping ratio $\beta_{\mathrm{s}}=\beta_{\mathrm{p}}=0.0025 \mathrm{in}$ both deviatoric and volumetric deformation. The $\mathrm{P}$-wave is characterized by a dimensionless frequency $\bar{f}_{\mathrm{p}}=k_{\mathrm{p}} a / \pi=2 a / \lambda_{\mathrm{p}}$, where $k_{\mathrm{p}}$ and $\lambda_{\mathrm{p}}$ denote the dilatational wavenumber and wavelength, respectively. A BE mesh consisting of 19021 eight node quadrilateral boundary elements with nodal collocation is used to discretize the canyon. The same values for the parameters $\eta, N_{\min }, \varepsilon$ and the tolerance in the GMRES solver as specified in subsection 4.1 are used in the BE method based on $\mathscr{H}$-matrices.

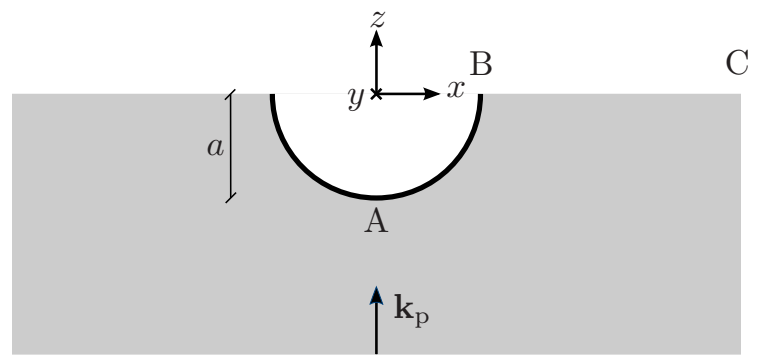

Figure 13: Semi-spherical canyon subjected to a vertically incident plane P-wave.

A subdomain formulation $[55,56]$ is employed, where the total wavefield is decomposed into an incident and scattered wavefield. Figure 14 and 15 show the modulus of the resulting horizontal and vertical displacements $\hat{u}_{x}(\mathbf{x}, \omega)$ and $\hat{u}_{z}(\mathbf{x}, \omega)$ along the path ABC (indicated on figure 13) as a function of the normalized horizontal distance $x / a$, at dimensionless frequencies $\bar{f}_{\mathrm{p}}=1 / 4$ and $\bar{f}_{\mathrm{p}}=1 / 2$, respectively. The displacements would be vertical with an amplitude of $2 \mathrm{~m} / \mathrm{Hz}$ in the absence of the cavity. It is clearly illustrated in these figures that a mode conversion takes place due to the presence of the cavity, however, 
resulting in significant horizontal displacements; the latter are zero at $x / a=0$ due to the symmetry of the problem. The results obtained with the BE method based on $\mathscr{H}$-matrices are compared to the solution of Sohrabi-Bidar et al. [51] and are in excellent agreement. Similar results have been obtained, amongst others, by Sánchez-Sesma [52], Reinoso et al. [53] and Chaillat et al. [54].

(a)

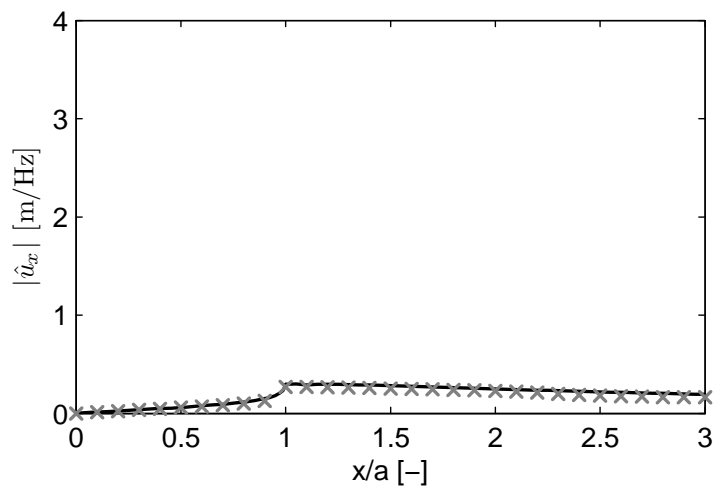

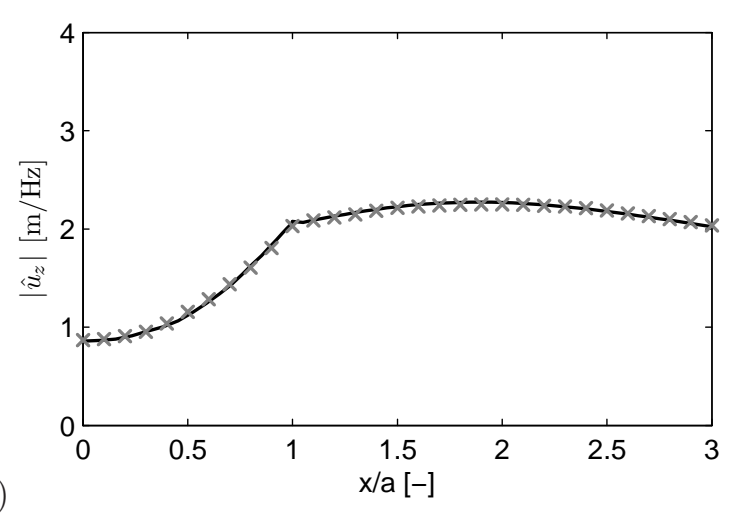

(b)

Figure 14: Modulus of the (a) horizontal and (b) vertical displacement $\hat{u}_{x}(\mathbf{x}, \omega)$ and $\hat{u}_{z}(\mathbf{x}, \omega)$ along the path $\mathrm{ABC}$ (indicated on figure 13) due to a vertically incident plane $\mathrm{P}$-wave at a dimensionless frequency $\bar{f}_{\mathrm{p}}=1 / 4$. The solution obtained with the hierarchical BE method (black lines) is compared to the solution of Sohrabi-Bidar et al. [51] (grey crosses).

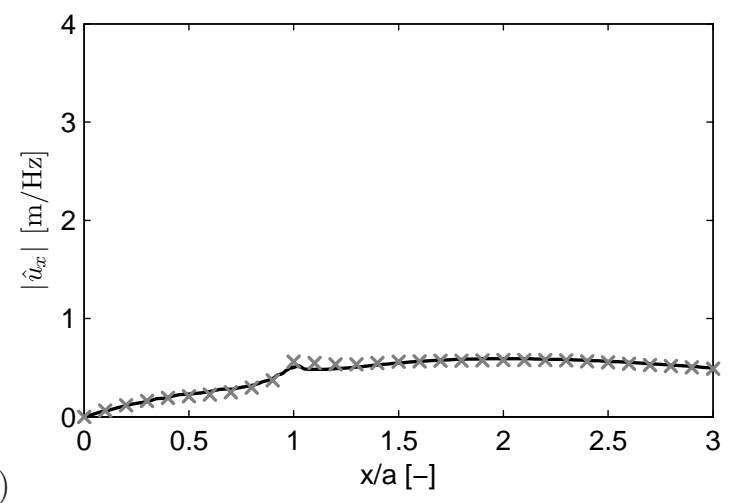

(a)

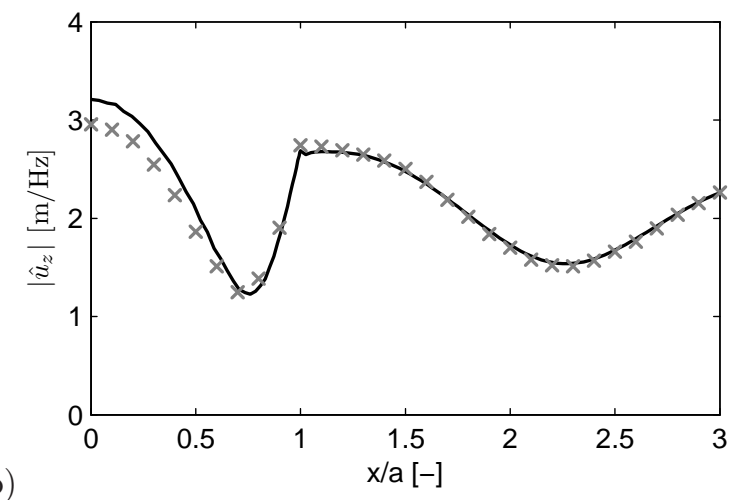

Figure 15: Modulus of the (a) horizontal and (b) vertical displacement $\hat{u}_{x}(\mathbf{x}, \omega)$ and $\hat{u}_{z}(\mathbf{x}, \omega)$ along the path ABC (indicated on figure 13) due to a vertically incident plane P-wave at a dimensionless frequency $\bar{f}_{\mathrm{p}}=1 / 2$. The solution obtained with the hierarchical BE method (black lines) is compared to the solution of Sohrabi-Bidar et al. [51] (grey crosses).

\section{Application: through-soil coupling of closely spaced structures}

It has been illustrated in the previous sections that the use of $\mathscr{H}$-matrices in the BE method combined with Green's functions for a horizontally layered halfspace results in a significant reduction of memory and CPU requirements, allowing to perform large scale BE computations. Furthermore, the method is also suited to tackle visco-elastodynamic problems, as illustrated in subsections 4.2 and 4.3 . The proposed methodology hence offers perspectives to model larger problems involving wave propagation in a layered halfspace, with possible applications in seismology, railway induced vibrations and dynamic soil-structure interaction.

One field of application is the numerical prediction of railway induced vibrations in an urban environment. While the interaction between the soil and multiple structures in dense urban areas subjected to seismic 
excitation has already been examined in a low frequency range $(<10 \mathrm{~Hz})[57,58,59]$, the numerical prediction of railway induced vibrations involves much higher frequencies up to $80 \mathrm{~Hz}$ [60]. As the number of boundary elements strongly increases with frequency, classical BE models only allow to take into account throughsoil coupling of very few structures and are unable to rigorously model wave propagation in dense urban areas characterized by many closely spaced structures. It is expected that structures close to the source of excitation will shield the surrounding buildings from the incident waves. In this section, the applicability of the proposed BE method based on $\mathscr{H}$-matrices is illustrated by means of a case study where the dynamic interaction between multiple buildings is investigated.

\subsection{Model description}

The case study considers a set of 12 identical masonry buildings resting on a layered halfspace (figure 16a). The same layered soil profile as introduced in subsection 4.2 is used. Figure 17 shows the frequencywavenumber spectrum of the vertical free field velocity $i \omega \tilde{u}_{z}\left(C_{r}, \omega\right)$ due to vertical harmonic excitation at the surface of the layered halfspace, presented in terms of the phase velocity $C_{r}=\omega / k_{r}$ instead of the wavenumber $k_{r}$. Peaks in the spectrum of $i \omega \tilde{u}_{z}\left(C_{r}, \omega\right)$ correspond to surface waves of the layered halfspace. The Rayleigh wave dispersion curves are superimposed on figure 17; four modes with associated cut-on frequencies exist in the frequency range considered. As the soil stiffness gradually increases with depth, the spectrum of the vertical free field velocity is dominated by the fundamental Rayleigh wave of the layered halfspace.
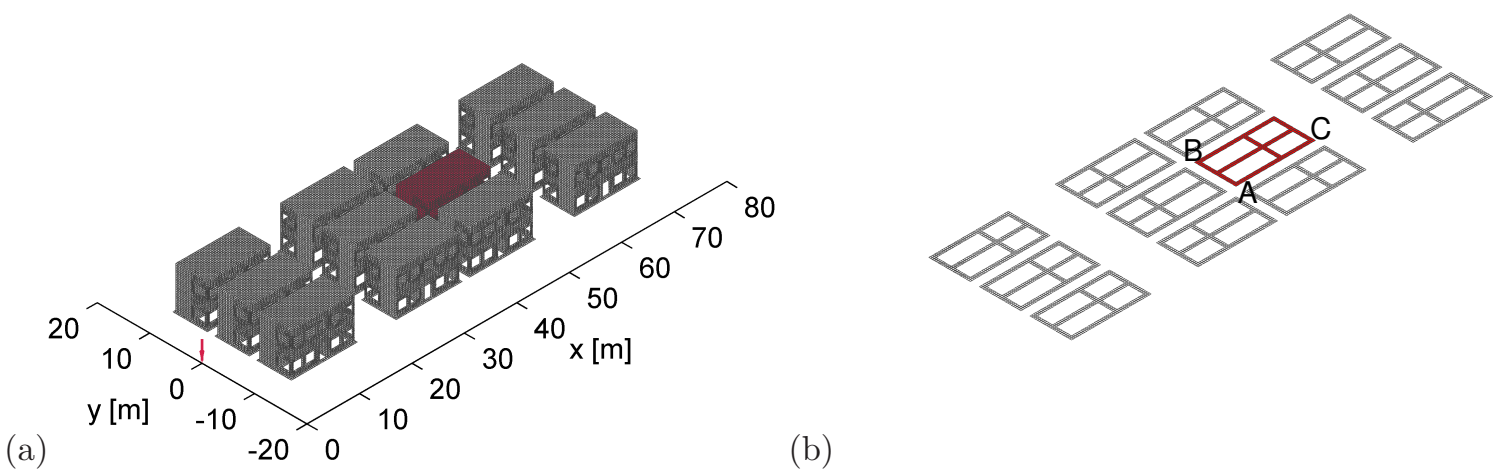

(b)

Figure 16: (a) Finite element and (b) boundary element mesh of the set of 12 masonry buildings.

Each masonry building has dimensions $12 \mathrm{~m} \times 6 \mathrm{~m} \times 8 \mathrm{~m}$ and has two stories, each subdivided into four rooms [61]. The interior and exterior walls have a thickness $t_{\mathrm{w}}=0.10 \mathrm{~m}$ and consist of clay brick masonry. The floors are concrete slabs with a thickness $t_{\mathrm{fl}}=0.20 \mathrm{~m}$. All floors are simply supported, corresponding to hinged joints at the slab edges. The structure is founded on a concrete strip foundation with a width $w_{\mathrm{f}}=0.60 \mathrm{~m}$ and a thickness $t_{\mathrm{f}}=0.20 \mathrm{~m}$. The buildings are modelled with the finite element method. The strip foundation, the walls and the floors are modelled by means of shell elements, using isotropic properties for the foundation and the floors and orthotropic properties for the masonry walls. The lintels above the door and the windows are modelled by means of beam elements. A detailed description of the single building model can be found in [61].

The 12 buildings are positioned in a symmetric layout with respect to the $x$-axis, with a separation distance of $2 \mathrm{~m}$ in the $y$-direction between the buildings. The front edges of four subsets of three buildings are aligned at $x=6 \mathrm{~m}, x=26 \mathrm{~m}, x=40 \mathrm{~m}$ and $x=60 \mathrm{~m}$, respectively.

The numerical prediction of railway induced vibrations in the built environment is a dynamic soilstructure interaction problem, coupling the source (railway track) and the receivers (buildings) through wave propagation in the soil $[60,62]$. In this case study, however, only the soil-structure interaction problem at 


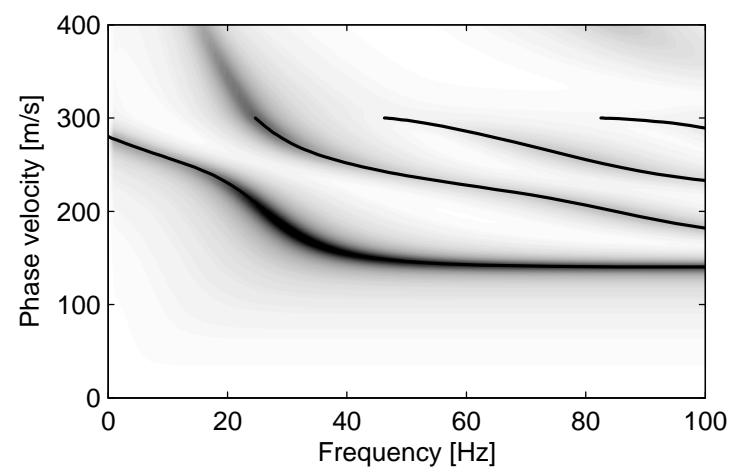

Figure 17: Frequency-wavenumber spectrum of the vertical free field velocity $i \omega \tilde{u}_{z}\left(C_{r}, \omega\right)$ due to vertical harmonic excitation at the surface of the layered halfspace. Superimposed are the Rayleigh wave dispersion curves of the first four modes.

the receiver side is addressed. The response of the buildings to an incident wavefield generated by a unit vertical point load acting on the surface of the layered halfspace at the origin of the coordinate system is therefore investigated, instead of an incident wavefield due to the passage of a train.

\subsection{Coupled FE-BE model}

A coupled FE-BE methodology in the frequency domain accounting for dynamic soil-structure interaction, based on a subdomain formulation $[55,56]$, is employed to calculate the response of the buildings to the incident wavefield. If $N$ structures are considered, a weak variational formulation of the equilibrium of structure $j(j=1, \ldots, N)$ results in the following set of coupled FE-BE equations:

$$
\left[\mathbf{K}_{j}+i \omega \mathbf{C}_{j}-\omega^{2} \mathbf{M}_{j}\right] \underline{\hat{\mathbf{u}}}_{j}(\omega)+\sum_{k=1}^{N} \hat{\mathbf{K}}_{j k}^{\mathrm{s}}(\omega) \underline{\hat{\mathbf{u}}}_{k}(\omega)=\underline{\hat{\mathbf{f}}}_{j}^{\mathrm{s}}(\omega) \quad \text { for } \quad j=1 \ldots N
$$

where $\underline{\hat{\mathbf{u}}}_{j}(\omega)$ collects the nodal degrees of freedom of structure $j$, while $\mathbf{K}_{j}, \mathbf{C}_{j}$ and $\mathbf{M}_{j}$ are the stiffness, damping and mass matrix of this structure. Rayleigh damping is assumed for the damping matrix $\mathbf{C}_{j}$ [61]. The diagonal blocks $(k=j)$ of the dynamic soil stiffness matrix $\hat{\mathbf{K}}_{j k}^{\mathrm{s}}(\omega)$ represent dynamic soilstructure interaction for structure $j$, while the off-diagonal blocks $(k \neq j)$ account for through-soil coupling of structures $j$ and $k$. The force vector $\underline{\hat{\mathbf{f}}}_{j}^{\mathrm{s}}(\omega)$ denotes the dynamic soil-structure interaction forces at the soil-structure interface $\Sigma_{j}$ due to the incident wavefield. A Craig-Bampton substructuring technique is furthermore used for every individual building, decomposing each structure $j$ into its foundation and superstructure.

The hierarchical BE method outlined in section 3 is employed to evaluate the dynamic soil stiffness matrices $\hat{\mathbf{K}}_{j k}^{\mathrm{s}}(\omega)$ and the force vectors $\underline{\mathbf{f}}_{j}^{\mathrm{s}}(\omega)$ arising from the incident wavefield. The free surface and the soil layers do not need to be discretized as Green's functions of a layered halfspace are employed. The finite elements of the foundations are coupled to a conforming boundary element mesh for the surrounding soil (figure 16b) and a nodal collocation scheme is used to facilitate the FE-BE coupling. As a nodal collocation scheme is used, the hierarchical clustering is based on the nodes rather than on the element centers. The same values for the parameters $\eta, N_{\min }, \varepsilon$ and the tolerance in the GMRES solver as specified in subsection 4.1 are used in the BE method based on $\mathscr{H}$-matrices (where $N_{\text {min }}$ indicates in this case a minimum number of nodes).

It also emphasized that a full 3D calculation is performed, without introducing additional assumptions concerning the lay-out of the buildings (i.e. no periodicity considerations are taken into account).

\subsection{Numerical results}

First, the response of the set of 12 buildings to an incident wavefield generated by a unit vertical harmonic point load at $10 \mathrm{~Hz}$ is considered. At this frequency, only one Rayleigh wave exists (figure 17); the 
Rayleigh wavelength in the soil equals $\lambda_{\mathrm{R}}(f)=C_{\mathrm{R}}(f) / f=25.7 \mathrm{~m}$. The incident wavefield, characterized by cylindrical wave fronts, is shown in figure 18a. Figure 18b shows the wavefield in the soil in case the presence of all 12 buildings is simultaneously taken into account. As the Rayleigh wavelength is larger than the dimensions of the strip foundations, the wavefield remains nearly cylindrical.
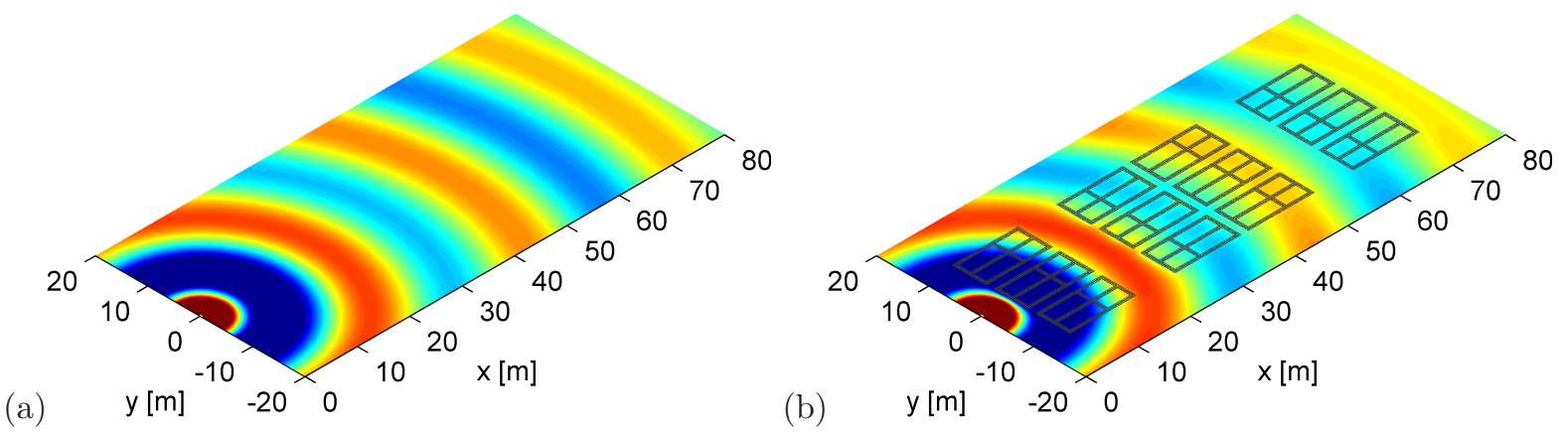

Figure 18: Real part of the vertical soil displacement $\hat{u}_{z}(\mathbf{x}, \omega)$ at $10 \mathrm{~Hz}$ (a) without and (b) with accounting for the presence of the buildings.

The influence of through-soil coupling on the structural response is illustrated in figure 19. Figure 19a shows the vertical displacement $\hat{u}_{z}(\mathbf{x}, \omega)$ of all buildings, in case the presence of the surrounding buildings is neglected for each building. This is obtained by solving equation (14) $N$ times, in which the soil stiffness matrices $\hat{\mathbf{K}}_{j k}^{\mathrm{s}}(\omega)$ are not considered for $k \neq j$. Figure 19b illustrates the structural response in case the presence of all 12 buildings is simultaneously taken into account. Comparison of figures 19a and 19b indicates that the structural response is nearly identical for the three buildings closest to the source, while the response of the buildings further away from the source turns out to be more affected by the presence of the surrounding buildings.

(a)

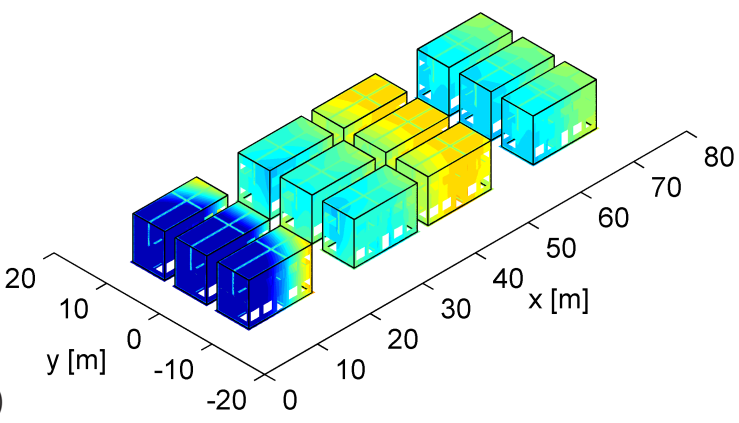

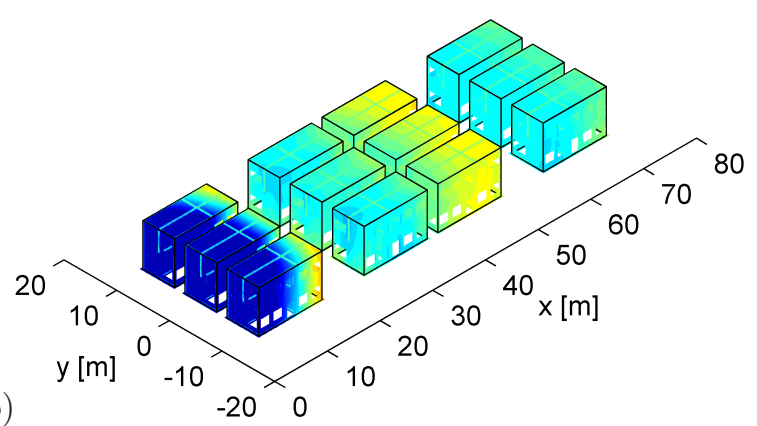

Figure 19: Real part of the vertical structural displacement $\hat{u}_{z}(\mathbf{x}, \omega)$ at $10 \mathrm{~Hz}$ (a) without and (b) with accounting for throughsoil coupling of the surrounding buildings.

The influence of through-soil coupling on the structural response is investigated in more detail for one particular building, which is indicated in red on figure 16. Figure 20 compares the modulus and phase of the vertical displacement $\hat{u}_{z}(\mathbf{x}, \omega)$ along the front wall-foundation edge AB. The response is not symmetrical due to the presence of doors and windows in the individual masonry buildings. While the variation of the displacements along this edge is similar in both cases, the amplitude is slightly reduced if throughsoil coupling is accounted for. This indicates that the buildings closer to the source shield the considered building from the incident wavefield. Furthermore, an almost constant phase shift of 0.65 rad is introduced along the edge $\mathrm{AB}$, as the Rayleigh wave fronts are slightly shifted due the presence of the buildings. Similarly, figure 21 compares the modulus and phase of the vertical displacement $\hat{u}_{z}(\mathbf{x}, \omega)$ along the side 
wall-foundation edge AC. The variation of the displacements along this edge is similar in both cases; a slight amplitude reduction and a phase shift of $0.65 \mathrm{rad}$ can be observed here as well.
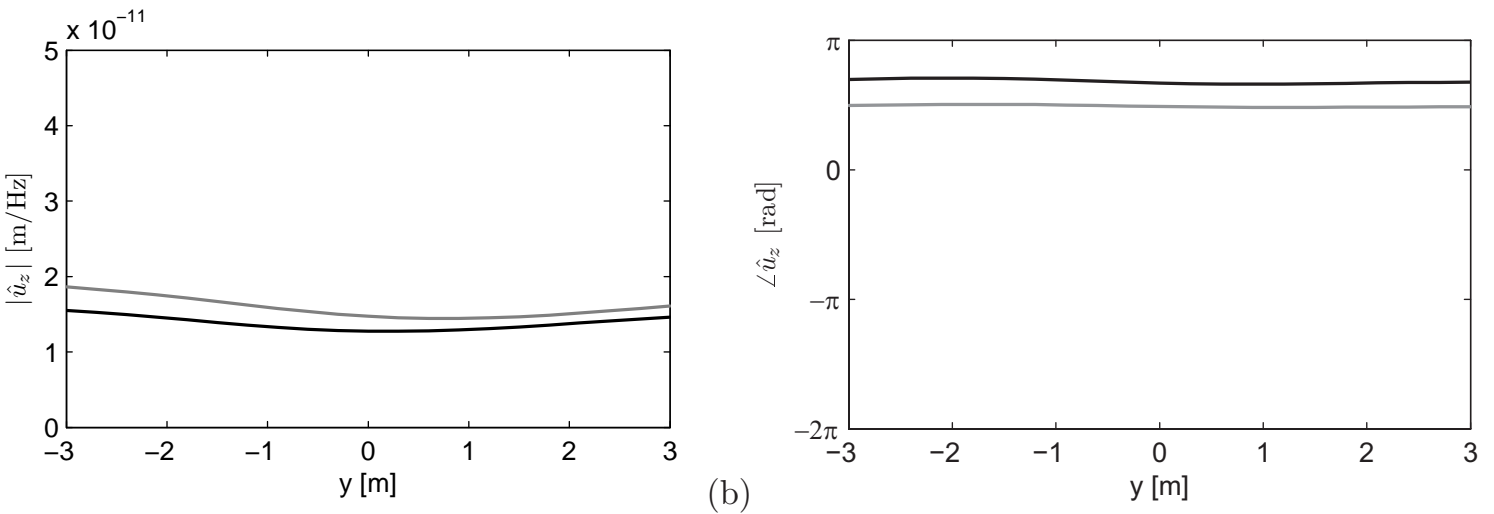

Figure 20: (a) Modulus and (b) phase of the vertical displacement $\hat{u}_{z}(\mathbf{x}, \omega)$ along the front wall-foundation edge $\mathrm{AB}$ at $10 \mathrm{~Hz}$ without (grey lines) and with (black lines) accounting for through-soil coupling of the surrounding buildings.

(a)

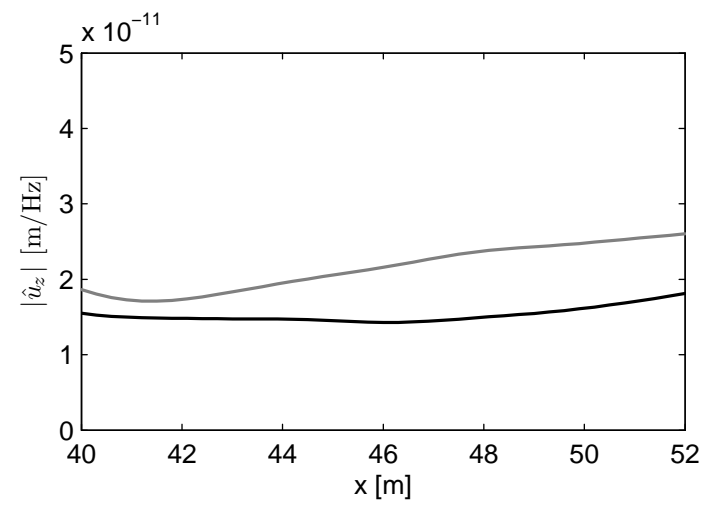

(b)

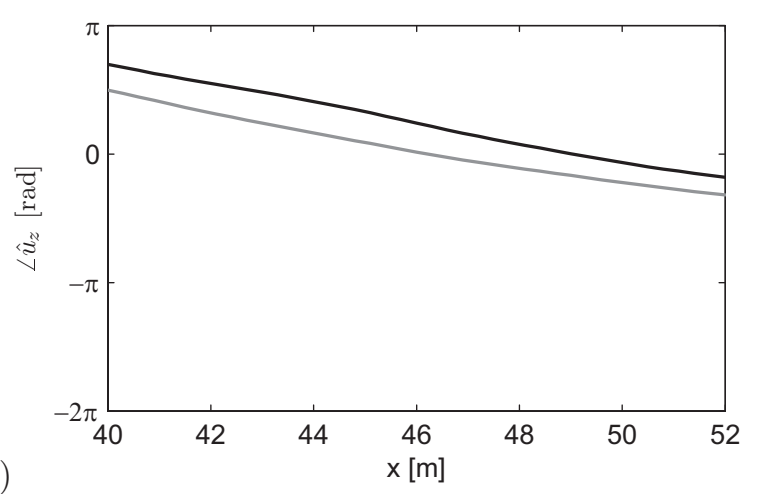

Figure 21: (a) Modulus and (b) phase of the vertical displacement $\hat{u}_{z}(\mathbf{x}, \omega)$ along the side wall-foundation edge AC at $10 \mathrm{~Hz}$ without (grey lines) and with (black lines) accounting for through-soil coupling of the surrounding buildings.

Second, the response of the set of 12 buildings to an incident wavefield generated by a unit vertical harmonic point load at a frequency of $50 \mathrm{~Hz}$ is considered. Although three modes exist at this frequency, the spectrum of the vertical free field velocity is still dominated by the fundamental Rayleigh wave (figure 17); the corresponding Rayleigh wavelength in the soil equals $\lambda_{\mathrm{R}}(f)=C_{\mathrm{R}}(f) / f=2.9 \mathrm{~m}$. The incident wavefield, characterized by cylindrical wave fronts, is shown in figure $22 \mathrm{a}$. The dynamic interaction between the buildings and the halfspace significantly changes the wavefield (figure 22b), as the wavelength in the soil has the same order of magnitude as the dimensions of the strip foundations.

The influence of through-soil coupling on the structural response is illustrated in figure 23. Figure 23a shows the vertical displacement $\hat{u}_{z}(\mathbf{x}, \omega)$ of all buildings, in case the presence of the surrounding buildings is neglected for each building, while figure 23b illustrates the structural response in case the presence of all 12 buildings is simultaneously taken into account. As in figure 18, the response of the three buildings closest to the source remains almost unaffected. The response of the other buildings, however, is considerably altered by the presence of the surrounding buildings.

The influence of through-soil coupling on the structural response is investigated in more detail for the same building as discussed before. Figures 24 and 25 compare the modulus and phase of the vertical dis- 

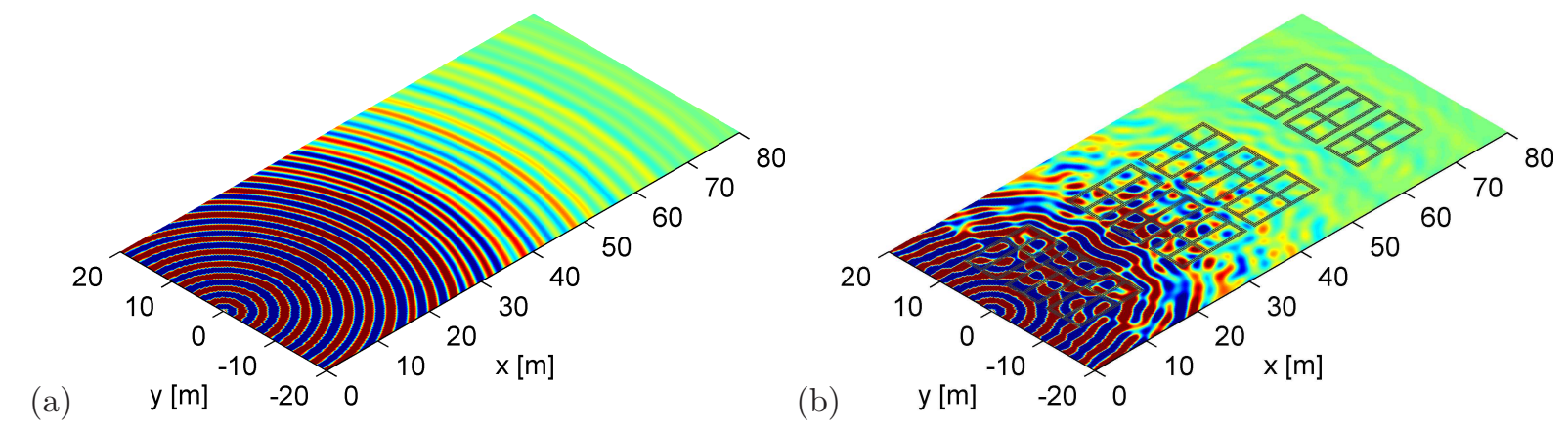

Figure 22: Real part of the vertical soil displacement $\hat{u}_{z}(\mathbf{x}, \omega)$ at $50 \mathrm{~Hz}$ (a) without and (b) with accounting for the presence of the buildings.
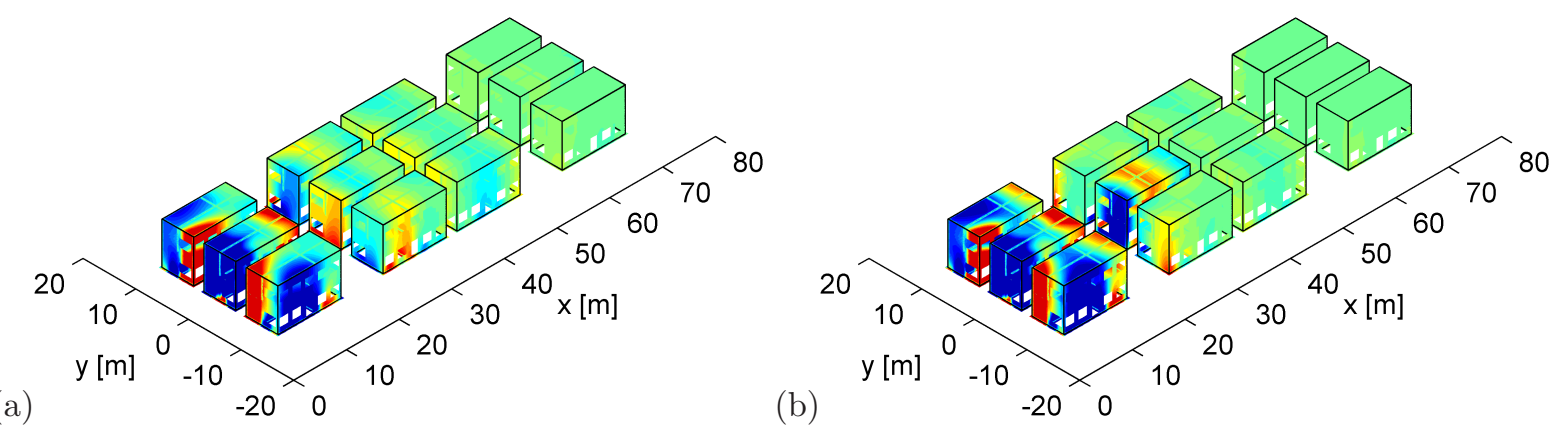

Figure 23: Real part of the vertical structural displacement $\hat{u}_{z}(\mathbf{x}, \omega)$ at $50 \mathrm{~Hz}$ (a) without and (b) with accounting for throughsoil coupling of the surrounding buildings. 
placement $\hat{u}_{z}(\mathbf{x}, \omega)$ along the front wall-foundation edge AB and side wall-foundation edge AC, respectively. It is clearly illustrated that the variation of vertical displacements along both edges strongly differs when through-soil coupling is accounted for.
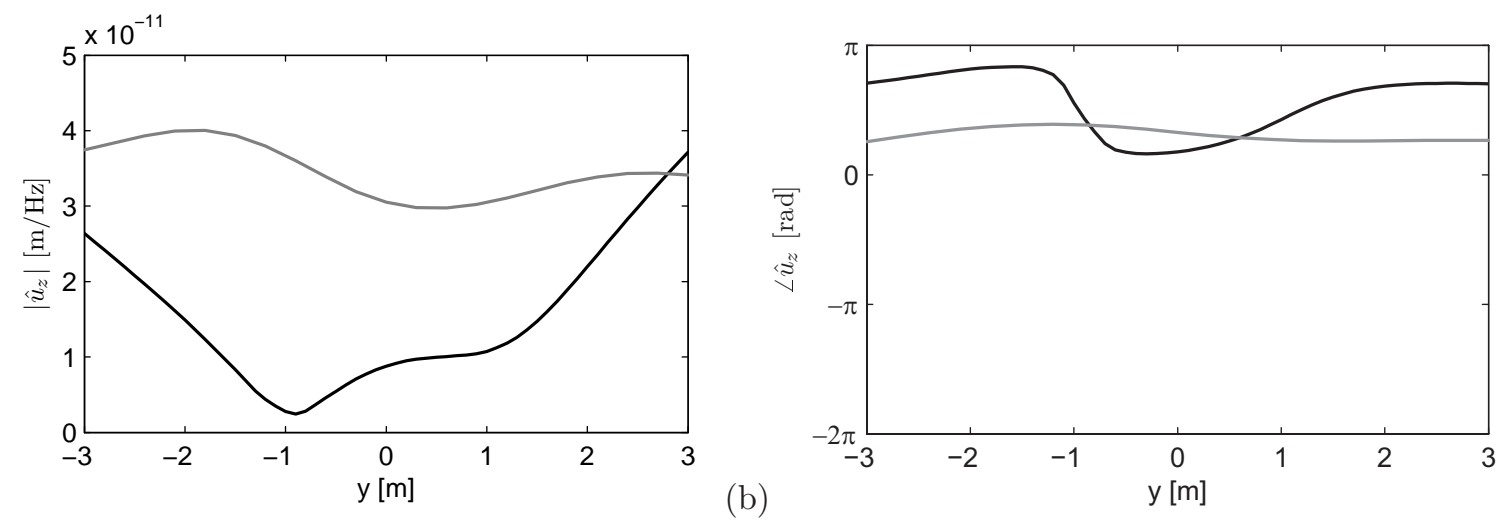

Figure 24: (a) Modulus and (b) phase of the vertical displacement $\hat{u}_{z}(\mathbf{x}, \omega)$ along the front wall-foundation edge $\mathrm{AB}$ at $50 \mathrm{~Hz}$ without (grey lines) and with (black lines) accounting for through-soil coupling of the surrounding buildings.
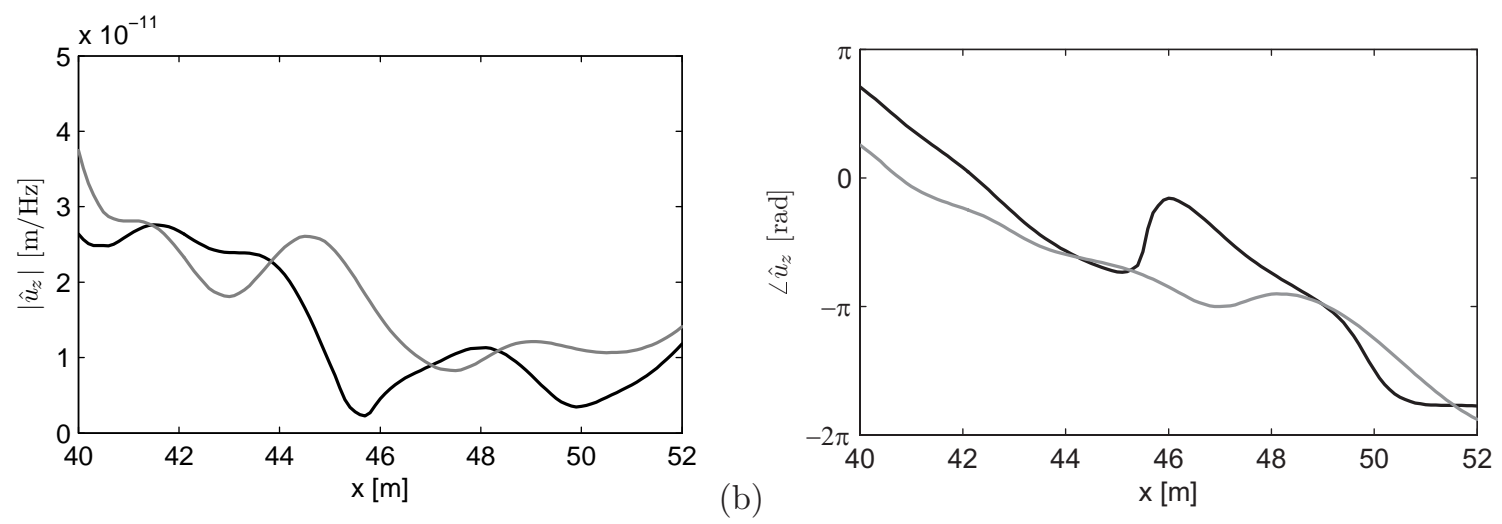

Figure 25: (a) Modulus and (b) phase of the vertical displacement $\hat{u}_{z}(\mathbf{x}, \omega)$ along the side wall-foundation edge AC at $50 \mathrm{~Hz}$ without (grey lines) and with (black lines) accounting for through-soil coupling of the surrounding buildings.

This case study indicates that wave propagation in the soil and the structural response are considerably affected in an urban environment. At low frequencies, this predominantly results in a shielding effect, reducing the amplitudes of the displacements, without drastically altering the wavefield. At higher frequencies, however, the wavelength in the soil becomes comparable to the foundation dimensions and the dynamic interaction between the buildings and the halfspace significantly changes the wavefield. It should furthermore be emphasized that this case study could not have been investigated as efficiently with existing BE formulations.

\section{Conclusions}

In this paper, the application of hierarchical matrices to boundary element methods based on Green's functions for a horizontally layered halfspace has been presented. The Green's functions are numerically computed by means of the direct stiffness method, as no closed form analytical expressions are available. 
Incorporating these Green's functions is advantageous, as there is no need to discretize the free surface and the layer interfaces. The ACA algorithm is used to compute low rank approximations of blocks corresponding to admissible cluster pairs, while an iterative method is employed to solve the resulting set of $\mathscr{H}-\mathrm{BE}$ equations.

The numerical implementation of the novel methodology has been validated and it has been demonstrated that although a compromise between accuracy and efficiency should be made, it is very efficient, very fast and sufficiently accurate. The method enables the fast evaluation of much larger boundary element models than before and is hence a valuable tool for researchers and engineers dealing with problems involving elastodynamic wave propagation in a stratified halfspace, providing the possibility to investigate complex problems in seismology and dynamic soil-structure interaction. The applicability of the method has clearly been illustrated by means of a case study that could not have been solved as efficiently with existing BE formulations. The dynamic interaction between closely spaced structures was investigated and it was illustrated that through-soil coupling of buildings can significantly alter the structural response, especially at higher frequencies.

\section{Acknowledgements}

The first author is a doctoral fellow and the second author is a postdoctoral fellow of the Research Foundation Flanders (FWO). The financial support is gratefully acknowledged.

\section{References}

[1] J.-F. Semblat, A. Duval, P. Dangla, Seismic site effects in a deep alluvial basin: numerical analysis by the boundary element method, Computers and Geotechnics 29 (7) (2002) 573-585.

[2] G. Lombaert, G. Degrande, D. Clouteau, Numerical modelling of free field traffic induced vibrations, Soil Dynamics and Earthquake Engineering 19 (7) (2000) 473-488.

[3] D. Beskos, T. Krauthammer, I. Vardoulakis (Eds.), Dynamic soil-structure interaction, A.A. Balkema, 1984.

[4] V. Rokhlin, Rapid solution of integral equations of classical potential theory, Journal of Computational Physics 60 (1985) $187-207$.

[5] W. Hackbusch, Z. Nowak, On the fast matrix multiplication in the boundary element method by panel clustering, Numerische Mathematik 54 (1989) 463-491.

[6] W. Hackbusch, A sparse matrix arithmetic based on $\mathscr{H}$-matrices. Part I: Introduction to $\mathscr{H}$-matrices, Computing 62 (2) (1999) 89-108.

[7] N. Nishimura, Fast multipole accelerated boundary integral equation methods, Applied Mechanics Reviews 22 (2002) $175-181$.

[8] H. Fujiwara, The fast multipole method for solving integral equations of three-dimensional topography and basin problems, Geophysical Journal International 140 (1) (2000) 198-210.

[9] S. Chaillat, M. Bonnet, J.-F. Semblat, A new fast multi-domain BEM to model seismic wave propagation and amplification in 3D geological structures, Geophysical Journal International 177 (2009) 509-531.

[10] E. Grasso, S. Chaillat, M. Bonnet, J.-F. Semblat, Application of the multi-level time-harmonic fast multipole BEM to 3-D visco-elastodynamics, Engineering Analysis with Boundary Elements 36 (5) (2012) 744-758.

[11] S. Chaillat, M. Bonnet, Formulation and fast evaluation of the multipole expansions of the elastic half-space fundamental solutions, 8th European Solid Mechanics Conference, Graz, Austria (July 2012).

[12] S. Rjasanow, O. Steinbach, The Fast Solution of Boundary Integral Equations (Mathematical and Analytical Techniques with Applications to Engineering), Springer-Verlag New York, 2007.

[13] M. Bebendorf, Hierarchical Matrices: A Means to Efficiently Solve Elliptic Boundary Value Problems, 1st Edition, Springer Publishing Company, 2008.

[14] L. Banjai, W. Hackbusch, Hierarchical matrix techniques for low- and high-frequency Helmholtz problems, IMA Journal of Numerical Analysis 28 (1) (2008) 46-79.

[15] J. Ostrowski, Z. Andjelić, M. Bebendorf, B. Cranganu-Cretu, J. Smajić, Fast BEM-Solution of Laplace Problems with $\mathcal{H}$-Matrices and ACA, IEEE Transactions on Magnetics 42 (4) (2006) 627-630.

[16] M. Bebendorf, R. Grzhibovskis, Accelerating Galerkin BEM for linear elasticity using adaptive cross approximation, Mathematical Methods in the Applied Sciences 29 (14) (2006) 1721-1747.

[17] F. Maerten, Adaptive cross-approximation applied to the solution of system of equations and post-processing for 3D elastostatic problems using the boundary element method, Engineering Analysis with Boundary Elements 34 (5) (2010) 483-491.

[18] M. Messner, M. Schanz, An accelerated symmetric time-domain boundary element formulation for elasticity, Engineering Analysis with Boundary Elements 34 (11) (2010) 944-955. 
[19] I. Benedetti, M. Aliabadi, A fast hierarchical dual boundary element method for three-dimensional elastodynamic crack problems, International Journal for Numerical Methods in Engineering 84 (9) (2010) 1038-1067.

[20] A. Milazzo, I. Benedetti, M. Aliabadi, Hierarchical fast BEM for anisotropic time-harmonic 3-D elastodynamics, Computers and Structures 96-97 (2012) 9-24.

[21] F. Rizzo, D. Shippy, An application of the correspondence principle of linear viscoelasticity theory, SIAM Journal on Applied Mathematics 21 (2) (1971) 321-330.

[22] L. Wheeler, E. Sternberg, Some theorems in classical elastodynamics, Archive for Rational Mechanics and Analysis 31 (1968) 51-90.

[23] J. Domínguez, Boundary elements in dynamics, Computational Mechanics Publications and Elsevier Applied Science, Southampton, United Kingdom, 1993.

[24] M. Bonnet, Boundary integral equation methods for solids and fluids, John Wiley and Sons, Chichester, United Kingdom, 1995.

[25] F. Rizzo, D. Shippy, M. Rezayat, A boundary integral equation method for radiation and scattering, International Journal for Numerical Methods in Engineering 21 (1985) 115-129.

[26] D. Aubry, D. Clouteau, A regularized boundary element method for stratified media, in: G. Cohen, L. Halpern, P. Joly (Eds.), Proceedings of the First International Conference on Mathematical and Numerical Aspects of Wave Propagation Phenomena, SIAM, Philadephia, Strasbourg, France, 1991, pp. 660-668.

[27] E. Kausel, J. Roësset, Stiffness matrices for layered soils, Bulletin of the Seismological Society of America 71 (6) (1981) $1743-1761$.

[28] M. Schevenels, S. François, G. Degrande, EDT: An ElastoDynamics Toolbox for MATLAB, Computers \& Geosciences 35 (8) (2009) 1752-1754.

[29] J. Talman, Numerical Fourier and Bessel transforms in logarithmic variables, Journal of Computational Physics 29 (1) (1978) 35-48.

[30] M. Schevenels, The impact of uncertain dynamic soil characteristics on the prediction of ground vibrations, Ph.D. thesis, Department of Civil Engineering, KU Leuven (2007).

[31] E. Pan, Static Green's functions in multilayered half spaces, Applied Mathematical Modelling 21 (8) (1997) 509-521.

[32] W. Thomson, Transmission of elastic waves through a stratified solid medium, Journal of Applied Physics 21 (1950) 89-93.

[33] N. Haskell, The dispersion of surface waves on multilayered media, Bulletin of the Seismological Society of America 73 (1953) 17-43.

[34] J. Dunkin, Computation of modal solutions in layered, elastic media at high frequencies, Bulletin of the Seismological Society of America 55 (2) (1965) 335-358.

[35] J. Luco, R. Apsel, On the Green's functions for a layered half-space. Part I, Bulletin of the Seismological Society of America 4 (1983) 909-929.

[36] R. Apsel, J. Luco, On the Green's functions for a layered half-space. Part II, Bulletin of the Seismological Society of America 73 (4) (1983) 931-951.

[37] E. Kausel, Fundamental solutions in elastodynamics: a compendium, Cambridge University Press, New York, 2006.

[38] L. Grasedyck, W. Hackbusch, Construction and arithmetics of $\mathscr{H}$-matrices, Computing 70 (2003) $295-334$.

[39] S. Börm, L. Grasedyck, W. Hackbusch, Introduction to hierarchical matrices with applications, Engineering Analysis with Boundary Elements 27 (5) (2003) 405-422.

[40] A. George, Nested dissection of a regular finite element mesh, SIAM Journal on Numerical Analysis 10 (1973) 345-363.

[41] L. Grasedyck, R. Kriemann, S. Le Borne, Domain decomposition based $\mathscr{H}-L U$ preconditioning, Numerische Mathematik 112 (4) (2009) 565-600.

[42] M. Bebendorf, Approximation of boundary element matrices, Numerische Mathematik 86 (2000) 565-589.

[43] M. Bebendorf, S. Rjasanow, Adaptive low-rank approximation of collocation matrices, Computing 70 (2003) 1-24.

[44] L. Grasedyck, Adaptive recompression of $\mathscr{H}$-matrices for BEM, Computing 74 (3) (2005) 205-223.

[45] M. Bebendorf, S. Kunis, Recompression techniques for adaptive cross approximation, Journal of Integral Equations and Applications 21 (3) (2009) 331-357.

[46] G. Golub, C. Van Loan, Matrix computations, 3rd Edition, John Hopkins University Press, Baltimore, MD, 1996.

[47] Y. Saad, M. Schultz, GMRES: a generalized minimal residual algorithm for solving nonsymmetric linear systems, SIAM Journal on Scientific and Statistical Computing 7 (3) (1986) 856-869.

[48] S. François, M. Schevenels, G. Degrande, BEMFUN: MATLAB toolbox for boundary elements in elastodynamics. Version 2.1 Build 16, User's guide BWM-2009-26, Department of Civil Engineering, KU Leuven (December 2009).

[49] G. Gazetas, Analysis of machine foundation vibrations: state of the art, Soil Dynamics and Earthquake Engineering 2 (1) (1983) 2-42.

[50] M. Bebendorf, Hierarchical $L U$ decomposition based preconditioners for BEM, Computing 74 (2005) $225-247$.

[51] A. Sohrabi-Bidar, M. Kamalian, M. K. Jafari, Time-domain BEM for three-dimensional site response analysis of topographic structures, International Journal for Numerical Methods in Engineering 79 (12) (2009) 1467-1492.

[52] F. Sánchez-Sesma, Diffraction of elastic waves by three-dimensional surface irregularities, Bulletin of the Seismological Society of America 73 (6) (1983) 1621-1636.

[53] E. Reinoso, L. Wrobel, H. Power, Three-dimensional scattering of seismic waves from topographical structures, Soil Dynamics and Earthquake Engineering 16 (1) (1997) 41-61.

[54] S. Chaillat, M. Bonnet, J.-F. Semblat, A multi-level fast multipole BEM for 3-D elastodynamics in the frequency domain, Computer Methods in Applied Mechanics and Engineering 197 (49-50) (2008) 4233-4249.

[55] D. Aubry, D. Clouteau, A subdomain approach to dynamic soil-structure interaction, in: V. Davidovici, R. Clough (Eds.), Recent advances in Earthquake Engineering and Structural Dynamics, Ouest Editions/AFPS, Nantes, 1992, pp. 251-272. 
[56] D. Aubry, D. Clouteau, G. Bonnet, Modelling of wave propagation due to fixed or mobile dynamic sources, in: N. Chouw, G. Schmid (Eds.), Workshop Wave '94, Wave propagation and Reduction of Vibrations, Ruhr Universität Bochum, Germany, 1994, pp. 109-121.

[57] D. Clouteau, D. Aubry, Modification of the ground motion in dense urban areas, Journal of Computational Acoustics 9 (4) (2001) 1659-1675.

[58] M. Kham, J.-F. Semblat, P.-Y. Bard, P. Dangla, Seismic city-site interaction: main governing phemomena through simplified numerical models, Bulletin of the Seismological Society of America 96 (5) (2006) 1934-1951.

[59] G. Lombaert, D. Clouteau, The resonant multiple wave scattering in the seismic response of a city, Waves in Random and Complex Media 16 (3) (2006) 205-230.

60] G. Lombaert, G. Degrande, J. Kogut, S. Francois, The experimental validation of a numerical model for the prediction of railway induced vibrations, Journal of Sound and Vibration 297 (3-5) (2006) 512-535.

[61] S. François, C. Karg, W. Haegeman, G. Degrande, A numerical model for foundation settlements due to deformation accumulation in granular soils under repeated small amplitude dynamic loading, International Journal for Numerical and Analytical Methods in Geomechanics 34 (3) (2010) 273-296.

[62] X. Sheng, C. Jones, D. Thompson, A theoretical model for ground vibration from trains generated by vertical track irregularities, Journal of Sound and Vibration 272 (3-5) (2004) 937-965. 